\title{
X-ray irradiation of the winds in binaries with massive components ${ }^{\star}$
}

\author{
J. Krtička ${ }^{1}$, J. Kubát ${ }^{2}$, and I. Krtičková ${ }^{1}$ \\ 1 Ústav teoretické fyziky a astrofyziky, Masarykova univerzita, Kotlářská 2, 61137 Brno, Czech Republic \\ e-mail: krticka@physics.muni.cz \\ 2 Astronomický ústav, Akademie věd České republiky, Fričova 298, 25165 Ondřejov, Czech Republic
}

Received 10 January 2015 / Accepted 20 April 2015

\begin{abstract}
Context. Binaries with hot massive components are strong X-ray sources. Besides the intrinsic X-ray emission of individual binary members originating in their winds, $\mathrm{X}$-ray emission stems from the accretion on the compact companion or from wind collision. Since hot star winds are driven by the light absorption in the lines of heavier elements, wind acceleration is sensitive to the ionization state. Therefore, the over-ionization induced by external X-ray source strongly influences the winds of individual components.

Aims. We studied the effect of external X-ray irradiation on hot star winds.

Methods. We used our kinetic equilibrium (NLTE) wind models to estimate the influence of external X-ray ionization for different $\mathrm{X}$-ray luminosities and source distances. The models are calculated for parameters typical of $\mathrm{O}$ stars.

Results. The influence of X-rays is given by the X-ray luminosity, by the optical depth between a given point and the X-ray source, and by a distance to the X-ray source. Therefore, the results can be interpreted in the diagrams of X-ray luminosity vs. the optical depth parameter. X-rays are negligible in binaries with low X-ray luminosities or at large distances from the X-ray source. The influence of X-rays is stronger for higher X-ray luminosities and in closer proximity of the X-ray source. There is a forbidden area with high $\mathrm{X}$-ray luminosities and low optical depth parameters, where the X-ray ionization leads to wind inhibition. There is excellent agreement between the positions of observed stars in these diagrams and our predictions. All wind-powered high-mass X-ray binary primaries lie outside the forbidden area. Many of them lie close to the border of the forbidden area, indicating that their X-ray luminosities are self-regulated. We discuss the implications of our work for other binary types.

Conclusions. X-rays have a strong effect on the winds in binaries with hot components. The magnitude of the influence of X-rays can be estimated from the position of a star in the diagram of X-ray luminosity vs. the optical depth parameter.
\end{abstract}

Key words. stars: winds, outflows - stars: mass-loss - stars: early-type - hydrodynamics

\section{Introduction}

Binary stars with hot massive components are well-known X-ray sources. A high-mass X-ray binary (HMXB) is a system consisting of a massive luminous hot star and a compact object (either a neutron star or a black hole). In these systems a fraction of the primary star matter, coming from either a hot star wind or a Roche-lobe overflow, is trapped in the gravitational well of the compact object (Davidson \& Ostriker 1973; Lamers et al. 1976). These objects belong to the most powerful stellar X-ray sources with highest X-ray luminosities $L_{\mathrm{X}} \approx 10^{37}-10^{38} \mathrm{erg} \mathrm{s}^{-1}$.

$\mathrm{Be} / \mathrm{X}$-ray binaries also constitute a hot star donor and a compact companion, however, the matter is accreted from the circumstellar disk of the Be-star primary in these objects (see Reig 2011, for a review). The X-ray luminosities of Be/X-ray binaries may reach up to $L_{X} \approx 10^{37} \mathrm{erg} \mathrm{s}^{-1}$. Finally, the X-ray emission may originate in the wind collision in binaries with non-degenerate hot components (Prilutskii \& Usov 1976; Cooke et al. 1978; Pittard 2009). This kind of interaction results in relatively weaker X-ray sources with $L_{\mathrm{X}} \approx 10^{32}-10^{33} \mathrm{erg} \mathrm{s}^{-1}$ (e.g., Sana et al. 2006b; Antokhin et al. 2008).

The hydrodynamics of the circumstellar matter in binary systems is very complex and, in fact, constitutes a time-dependent

* Figures 3-8 are available in electronic form at http://www . aanda.org problem of radiative hydrodynamics (Friend \& Castor 1982; Blondin et al. 1990; Feldmeier et al. 1996). Therefore, most studies concentrate on the dynamics of the matter leading to the $\mathrm{X}$-ray generation. However, there is also a feedback effect of the produced X-rays on the stellar wind of hot component(s).

The feedback effect is connected with X-ray photoionization of the stellar wind (MacGregor \& Vitello 1982; van Loon et al. 2001; Watanabe et al. 2006). Because the stellar wind of hot stars is driven by light absorption in lines of heavier elements (e.g., carbon, nitrogen, and iron), photoionization may affect wind driving (Watanabe et al. 2006). This may even result in wind stagnation and possibly fall back on the source star (Krtička et al. 2012).

The problem of the X-ray photoionization should be treated using 3D hydrodynamical simulations coupled with radiative transfer and statistical equilibrium equations. These kinds of calculations are likely beyond the possibilities of current computers. To make the problem more tractable, one either uses hydrodynamical simulations with a simplified form of the radiative force (e.g. Friend \& Castor 1982; Blondin et al. 1990; Feldmeier et al. 1996; Hadrava \& Čechura 2012; Parkin \& Sim 2013) or 1D stationary wind models that account for the influence of X-ray photoionization on the level populations (e.g., Krtička et al. 2012). Time dependent hydrodynamical simulations can explain the wind structure in binaries in detail, but they cannot 
predict for which binary parameters the effect of X-ray irradiation becomes important.

We concentrate on a detailed solution of statistical equilibrium equations and neglect the time-dependent phenomena. We provide a grid of stationary $1 \mathrm{D}$ wind models with external X-ray irradiation for a wide grid of $\mathrm{O}$ star parameters. The X-ray irradiation is parameterized by the corresponding X-ray luminosity and the distance of the X-ray source from the star with wind. The wind is only calculated in the direction towards the X-ray source. This approach is sufficient to infer the effect of X-ray irradiation on the radiative force and yields a reliable estimate of the wind velocity in the direction of the X-ray source.

\section{Influence of X-rays on the wind ionization}

The influence of the X-rays on the ionization state of the wind is stronger the lower the X-ray optical depth between the X-ray source and a given point in the wind. The radial optical depth between the X-ray source at radius $D$ and point at radius $r$ is given by

$\tau_{v}(r)=\left|\int_{r}^{D} \kappa_{v}\left(r^{\prime}\right) \rho\left(r^{\prime}\right) \mathrm{d} r^{\prime}\right|$,

with distances measured with respect to the centre of windlosing star. The absolute value in this equation ensures that the optical depth is positive even for $r>D$. The X-ray optical depth $\tau_{v}(r)$ depends on density, which follows from the continuity equation as $\rho(r)=\dot{M} /\left(4 \pi r^{2} v_{r}\right)$, where $\dot{M}$ and $v_{r}$ are the wind mass-loss rate and the radial velocity, respectively. Assuming that the X-ray mass-absorption coefficient $\kappa_{v}$ is spatially constant and that the wind already reached its terminal velocity $v_{\infty}$, Eq. (1) can be written as

$\tau_{v}(r) \approx \frac{\kappa_{v} \dot{M}}{4 \pi v_{\infty}}\left|\frac{1}{r}-\frac{1}{D}\right|$.

The wind opacity in the X-ray domain mostly stems from the direct and Auger ionization. Equation (2) may motivate one to plot stars in the $L_{X}-\tau_{v}$ diagram to estimate the influence of X-rays on the wind ionization state (Krtička et al. 2012, Fig. 4).

Inspired by Eq. (2), we introduce a non-dimensional X-ray optical depth parameter

$t_{\mathrm{X}}=\frac{\dot{M}}{v_{\infty}}\left(\frac{1}{R_{*}}-\frac{1}{D}\right)\left(\frac{10^{3} \mathrm{~km} \mathrm{~s}^{-1} 1 R_{\odot}}{10^{-8} M_{\odot} \mathrm{yr}^{-1}}\right)$

to characterize the radial optical depth between the stellar surface and the X-ray source. This parameter estimates the influence of X-rays on the ionization state in the region of the critical point where the wind mass-loss rate is determined. The critical point is defined as a point where the speed of the Abbott waves, the fastest waves in the wind, is equal to the wind velocity (Abbott 1980; Owocki \& Rybicki 1984). If the matter is highly overionized close to the critical point, then the wind is not accelerated radiatively since higher ions are not efficient wind drivers (e.g. Krtička et al. 2012). This leads to wind inhibition. Therefore, we expect a strong influence of X-rays in stars with large X-ray luminosity $L_{X}$ and low X-ray optical depth parameter $t_{\mathrm{X}}$, whereas we assume weak X-ray influence in stars with low $L_{\mathrm{X}}$ and high $t_{\mathrm{X}}$. For medium values of these parameters, the $\mathrm{X}$-rays may just affect the wind velocity and lead possibly to wind stagnation.

A description of the influence of X-ray irradiation on the wind state via the optical depth parameter $t_{\mathrm{X}}$ introduced in
Eq. (3) can only be used for stars with a similar ionization and density structure. For a more complete description of this problem, one also has to account for the $n^{2}$ dependence of the recombination that follows the X-ray ionization ( $n$ is the electron number density). Therefore, with the mean intensity of the radiation $J_{v}^{\mathrm{X}}$ at the distance $d=|D-r|$ from the X-ray source (see also Krtička et al. 2012, Eq. (4)),

$J_{v}^{\mathrm{X}}=\frac{L_{v}^{\mathrm{X}}}{16 \pi^{2} d^{2}} \mathrm{e}^{-\tau_{\nu}(r)}$,

one can introduce the ionization parameter

$\xi(r)=\frac{1}{n d^{2}} \int L_{\nu}^{\mathrm{X}} \mathrm{e}^{-\tau_{v}(r)} \mathrm{d} v$,

which estimates the influence of X-ray radiation on the ionization equilibrium of the wind. Here $L_{v}^{\mathrm{X}}$ is the $\mathrm{X}$-ray luminosity per unit of frequency and the integration goes over the X-ray domain. In the optically thin limit this gives the ionization parameter $\xi \sim L_{X} /\left(n d^{2}\right)$ introduced by Tarter et al. (1969; see also Hatchett \& McCray 1977), where $L_{\mathrm{X}}=\int L_{v}^{\mathrm{X}} \mathrm{d} v$.

\section{Description of the X-ray irradiated wind models}

For our calculations, we used spherically symmetric stationary wind models of Krtička \& Kubát (2010). The radiative force was calculated using the solution of the comoving frame (CMF) radiative transfer equation with occupation numbers derived from the kinetic equilibrium (NLTE) equations. The model enables us to predict the wind structure, including the wind mass-loss rate and terminal velocity, consistently from global stellar parameters only.

The wind ionization and excitation state was calculated from the NLTE equations. Ionic models for the NLTE calculations are based on the Opacity and Iron Project results (Seaton et al. 1992; Hummer et al. 1993) and on the data described by Pauldrach et al. (2001). Part of the ionic models was adopted from TLUSTY model atmosphere input files (Lanz \& Hubeny 2003, 2007). The line radiative force was derived using the radiative flux calculated from the CMF radiative transfer equation (Mihalas et al. 1975) with the actual occupation numbers calculated from the NLTE equations. The line opacity data used in the line force calculation were extracted from the VALD database (Piskunov et al. 1995; Kupka et al. 1999). The occupation numbers derived from the NLTE equations were also used to calculate the radiative cooling and heating terms (Kubát et al. 1999). The emergent surface flux (inner boundary condition) was taken from the H-He spherically symmetric NLTE model stellar atmospheres of Kubát (2003, and references therein). The resulting hydrodynamical equations, that is, the continuity equation, equation of motion (with CMF radiative force), and the energy equation (with radiative cooling and heating), were solved iteratively together with NLTE and radiative transfer equations to obtain the wind density, velocity, and temperature structure.

Because the velocity field may become non-monotonic in the presence of the external irradiation, we do not use the CMF procedure of the radiative force calculation directly. Instead, as in Krtička et al. (2012), we calculate the ratio of the CMF and Sobolev line force (see Krtička \& Kubát 2010) for a model without external X-ray irradiation and use this ratio to correct the Sobolev line force in the models with external X-ray irradiation. The numerical test showed that this approach introduces very minor difference (below 1\%) between models with CMF line force and models with scaled Sobolev line force. Moreover, we 
use the model atmosphere flux for the calculation of the Sobolev line force. Therefore, we neglect nonlocal radiative coupling between absorption zones, which occurs in the non-monotonic flows (Rybicki \& Hummer 1978; Feldmeier \& Nikutta 2006).

The influence of the secondary component is only taken into account by inclusion of external X-ray irradiation. This is supposed to originate in the wind accretion on the compact companion or in the wind-wind collision. The X-ray irradiation is modelled by an additional term in the mean intensity $J_{v}$ in the form of Eq. (4). The optical depth $\tau_{v}(r)$ along a radial ray in the direction of the X-ray source is given by Eq. (1). The frequency distribution of emergent $\mathrm{X}$-rays $L_{v}^{\mathrm{X}}$ is for simplicity approximated by the power law $L_{v}^{\mathrm{X}} \sim v^{-1}$ from 0.5 to $20 \mathrm{keV}$ (cf. Watanabe et al. 2006). The total X-ray luminosity $L_{\mathrm{X}}=\int L_{v}^{\mathrm{X}} \mathrm{d} v$ and the distance of the X-ray source $D$ are free parameters of the model grid.

The absorption coefficient and the density in Eq. (1) can be derived directly from the actual model. However, to simplify the calculation of the external irradiation term Eq. (4) in the presence of the kink in the velocity law (see below), for the calculation of $J_{v}^{\mathrm{X}}$ we use density and absorption coefficient in the form of

$\rho(r)=\frac{\dot{M}}{4 \pi r^{2} v(r)}$,

$v(r)=\min \left(\tilde{v}(r), v_{\text {kink }}\right)$,

$\kappa_{v}(r)=\tilde{\kappa}_{v}^{\mathrm{X}}$,

where $\tilde{\kappa}_{v}^{\mathrm{X}}$ is the depth-independent approximation of X-ray opacity, $v_{\text {kink }}$ is the velocity of the kink (see below) if it is present in the models, and otherwise $v_{\text {kink }}=\infty$. The fits to the wind velocity $\tilde{v}(r)$ and absorption coefficient $\tilde{\kappa}_{v}^{\mathrm{X}}$ are derived from the model with no external irradiation. The wind velocity is fitted as (Krtička \& Kubát 2011)

$$
\begin{aligned}
& \tilde{v}(r)=\left[v_{1}\left(1-\frac{R_{*}}{r}\right)+v_{2}\left(1-\frac{R_{*}}{r}\right)^{2}+v_{3}\left(1-\frac{R_{*}}{r}\right)^{3}\right] \\
& \times\left\{1-\exp \left[\gamma\left(1-\frac{r}{R_{*}}\right)^{2}\right]\right\},
\end{aligned}
$$

where $v_{1}, v_{2}, v_{3}$, and $\gamma$ are free parameters of the fit. The X-ray opacity per unit of mass averaged for radii $1.5 R_{*}-5 R_{*}$ is approximated as

$\log \left(\frac{\tilde{\kappa}_{v}^{\mathrm{X}}}{1 \mathrm{~cm}^{2} \mathrm{~g}^{-1}}\right)=\left\{\begin{array}{l}\min \left(a_{1} \log \lambda+b_{1}, \log a_{0}\right), \quad \lambda<\lambda_{1}, \\ a_{2} \log \lambda+b_{2}, \quad \lambda>\lambda_{1},\end{array}\right.$

where $\lambda_{1}=20.18$. The parameter $\lambda$ is non-dimensional, and has the same value as the wavelength in units of $\AA$. Here $a_{0}, a_{1}, b_{1}$, $a_{2}$, and $b_{2}$ are parameters of the fit.

The models were calculated in the direction towards the $\mathrm{X}$-ray source. The influence of the X-ray irradiation is strongest in this direction and it can be expected to decrease for the rays with increasing angular distance from the source.

\section{Calculated wind models}

The adopted grid of stellar parameters corresponds to $\mathrm{O}$ stars in the effective temperature range $30000-42500 \mathrm{~K}$. The stellar masses and radii in Table 1 were calculated from the effective temperature using empirical relations of Martins et al. (2005a). These are, together with the X-ray luminosity and the distance from the X-ray source, the parameters of the model grid. The $\mathrm{X}$-ray luminosities and the X-ray source distances were selected to correspond to typical hot star X-ray sources. Wind parameters
Table 1. Stellar parameters of the model grid.

\begin{tabular}{rccrc}
\hline \hline & Model & $\begin{array}{c}T_{\text {eff }} \\
{[\mathrm{K}]}\end{array}$ & $\begin{array}{r}R_{*} \\
{\left[R_{\odot}\right]}\end{array}$ & $\begin{array}{c}M \\
{\left[M_{\odot}\right]}\end{array}$ \\
\hline Supergiants (I) & $300-1$ & 30000 & 22.4 & 28.8 \\
& $375-1$ & 37500 & 19.8 & 48.3 \\
& $425-1$ & 42500 & 18.5 & 70.3 \\
\hline Main & $300-5$ & 30000 & 6.6 & 12.9 \\
sequence (V) & $375-5$ & 37500 & 9.4 & 26.8 \\
& $425-5$ & 42500 & 12.2 & 45.0 \\
\hline
\end{tabular}

Table 2. Parameters of the velocity law fits.

\begin{tabular}{rrrrr}
\hline \hline Model & $v_{1}\left[\mathrm{~km} \mathrm{~s}^{-1}\right]$ & $v_{2}\left[\mathrm{~km} \mathrm{~s}^{-1}\right]$ & $v_{3}\left[\mathrm{~km} \mathrm{~s}^{-1}\right]$ & $\gamma$ \\
\hline $300-1$ & 2280 & -680 & 0 & -610 \\
$375-1$ & 2700 & -980 & 0 & -6300 \\
$425-1$ & 2720 & 0 & -380 & -18600 \\
\hline $300-5$ & 2680 & -130 & 0 & -11700 \\
$375-5$ & 3380 & -1320 & 0 & -6900 \\
$425-5$ & 2770 & 0 & -510 & -13700 \\
\hline
\end{tabular}

Table 3. Parameters of the opacity fits.

\begin{tabular}{rrrrrr}
\hline \hline Model & $a_{0}$ & $a_{1}$ & $b_{1}$ & $a_{2}$ & $b_{2}$ \\
\hline $300-1$ & 215 & 2.619 & -0.917 & 2.655 & -1.478 \\
$375-1$ & 206 & 2.558 & -0.857 & 2.665 & -1.516 \\
$425-1$ & 191 & 2.482 & -0.794 & 2.620 & -1.530 \\
\hline $300-5$ & 210 & 2.618 & -0.917 & 2.649 & -1.466 \\
$375-5$ & 200 & 2.516 & -0.819 & 2.670 & -1.534 \\
$425-5$ & 197 & 2.497 & -0.804 & 2.647 & -1.537 \\
\hline
\end{tabular}

for the stars in Table 1 are given in Krtička \& Kubát (2012). The parameters of the velocity law fit Eq. (7) and opacity fits Eq. (8) are given in Tables 2 and 3, respectively.

The resulting wind models are given in Figs. 3-8. Here we plot the dependence of the radial wind velocity on radius in the wind models for individual studied model stars from Table 1 for different X-ray luminosities and X-ray source distances.

For a low X-ray luminosity $L_{X}$, or a large X-ray source distance $D$, the influence of the external $\mathrm{X}$-ray source on the wind model is only marginal. In this case the X-ray ionization influences only trace ionization states (via direct and Auger ionization, MacFarlane et al. 1994; Pauldrach et al. 1994). This modifies the emergent spectra because other trace ions with a high degree of ionization appear (Snow \& Morton 1976; Lamers \& Snow 1978), but the wind structure remains unaffected. Wind models for supergiant stars 375-1 and 425-1 with $L_{\mathrm{X}}=10^{31} \mathrm{erg} \mathrm{s}^{-1}$ (see Figs. 5 and 7) are examples of models with negligible influence of X-ray irradiation. Because new ionization states appear that may drive the wind, the wind terminal velocity may be slightly higher (Krtička \& Kubát 2009). For example, this explains why 300-1 model with $L_{\mathrm{X}}=10^{37} \mathrm{erg} \mathrm{s}^{-1}$ and $D=5000 R_{\odot}$ has higher terminal velocity than the same model, but with $D=7000 R_{\odot}$ (see Fig. 3).

For higher X-ray luminosities $L_{\mathrm{X}}$ (or a lower X-ray source distance $D$ ), the effect of $\mathrm{X}$-ray ionization becomes stronger. The ionization states that drive a wind in a case without X-ray irradiation become less populated close to the X-ray source, which results in a decrease of the radiative force and the wind velocity in the outer parts of the wind (e.g. 300-1 wind models with $L_{\mathrm{X}}=10^{38} \mathrm{erg} \mathrm{s}^{-1}$, Fig. 3). The decrease of the radiative force 
may be so strong that the accelerating wind solution is not possible any more, and the wind velocity switches to decelerating overloaded solutions (Feldmeier \& Shlosman 2000; Feldmeier et al. 2008) with a typical kink in the velocity profile. This leads to a non-monotonic velocity law and decrease in the terminal velocity, which were deduced from observations of HMXBs by Kaper et al. (1993) and calculated for Vela X-1 by Krtička et al. (2012). The decelerating regions of the kink may be followed by accelerating regions if the kink is weak (e.g. 300-1 wind models with $L_{\mathrm{X}}=10^{32} \mathrm{erg} \mathrm{s}^{-1}$ in Fig. 3 or $425-1$ models with $L_{\mathrm{X}}=10^{34} \mathrm{erg} \mathrm{s}^{-1}$ and $D \geq 25 R_{\odot}$ in Fig. 7). For stronger kinks, the switch back to the accelerating solution is missing (e.g. 375-5 models with $L_{X}=10^{35} \mathrm{erg} \mathrm{s}^{-1}$ in Fig. 6). However, our calculations simplify the treatment of the radiative force in the kink because we do not account for the complex line resonances that occur in this case (e.g. Feldmeier et al. 2008).

With decreasing source distance $D$ (or increasing X-ray luminosity $L_{\mathrm{X}}$ ) the kink position moves towards the stellar surface (see model 300-1 with $L_{X}=10^{34} \mathrm{erg} \mathrm{s}^{-1}$ in Fig. 3). If the kink occurs for velocities lower than the escape speed, the wind never leaves the star and the wind material falls back to the star (for example, this happens for the 300-1 model with $L_{X}=10^{37} \mathrm{erg} \mathrm{s}^{-1}$ and $D=200 R_{\odot}$ ). Our models only describe an accelerating part of the solution and the fall back has to be studied using time-dependent models (see Porter \& Skouza 1999, for a similar situation).

In the case when the kink reaches the critical point, the $\mathrm{X}$-rays start to significantly influence the wind mass-loss rate. The wind may be completely inhibited by the X-rays. In some cases, it is still possible to find a stationary solution (with the kink now being a critical point, not shown here), however, with a mass-loss rate typically lower by one or two orders of magnitude. Time-dependent models are needed to study this problem.

Derived results are summarized in the diagrams of $L_{X}$ vs. $t_{X}$ in Fig. 1. Each diagram indicates results for one of the model stars listed in Table 1 . For a given star (with fixed $\dot{M}$ and $v_{\infty}$ ), the optical depth parameter $t_{\mathrm{X}}$ in Eq. (3) only depends on the X-ray source distance $D$, and $t_{\mathrm{X}}$ is an increasing function of $D$. Wind models with a negligible influence of X-rays appear in the right bottom part of diagrams of $L_{\mathrm{X}}$ vs. $t_{\mathrm{X}}$ (high $t_{\mathrm{X}}$ and low $L_{\mathrm{X}}$ ). Wind models in which the $X$-ray source causes a decrease in terminal velocity (denoted by the $\times$ symbol) appear in a diagonal strip of the diagram of $L_{\mathrm{X}}$ vs. $t_{\mathrm{X}}$. A forbidden area, where the X-rays lead to wind inhibition, is located in the upper left corner of the diagrams of $L_{X}$ vs. $t_{X}$ diagrams.

The influence of the X-rays may be simply described by the ionization parameter $\xi$ introduced in Eq. (5). This is shown in Fig. 2, where we plot the positions of all studied models in the diagrams of $L_{X}$ vs. $\xi$. It follows that for low values of the ionization parameter, $\xi \lesssim 0.01 \mathrm{erg} \mathrm{s}^{-1} \mathrm{~cm}$, the X-rays may influence the wind ionization state, but their influence on wind structure is negligible. The X-rays significantly influence the winds for $\xi \gtrsim 0.01 \mathrm{erg} \mathrm{s}^{-1} \mathrm{~cm}$. Large ionization parameters $\xi \gtrsim 1-10 \mathrm{erg} \mathrm{s}^{-1} \mathrm{~cm}$ lead to the wind inhibition. These parameter values correspond to the forbidden area in the diagrams of $L_{\mathrm{X}}$ vs. $t_{\mathrm{X}}$.

The borderline between the ionization parameters that lead to wind inhibition and those that do not is not the same for all model stars in Fig. 2. There are different reasons for this, the most important is likely the influence of the energy distribution of the irradiating X-rays. The X-rays become harder with increasing optical depth between the source and a given point (because of frequency-dependent opacity, see Eq. (8)), leading to a smaller influence of the X-ray irradiation. Therefore, a distant obscured source has a smaller effect on wind ionization than a closer and weaker unobscured source with the same $\xi$. The wind optical depth in model $300-5$ is so low that there is no significant obscuration even for the distant sources. Consequently, the $\mathrm{X}$-rays already influence the wind for $\xi \approx 1 \mathrm{erg} \mathrm{s}^{-1} \mathrm{~cm}$.

\section{Comparison with observations}

\subsection{High-mass $X$-ray binaries}

The existence of the forbidden area in the diagram of $L_{X}$ vs. $t_{X}$ can be tested against the observed parameters of HMXBs. For this purpose we searched the literature for available parameters of wind-powered Galactic HMXBs (these are listed in Table 4). In this table we restricted ourselves to HMXBs with primary effective temperatures $T_{\text {eff }} \gtrsim 25000 \mathrm{~K}$ to avoid the bistability jump (Pauldrach \& Puls 1990; Vink et al. 1999), which may complicate the estimate of wind parameters for cooler stars and deserves special study. Our list of HMXBs with known parameters is likely incomplete, but it is a representative subset that characterizes the complete sample.

Stellar and binary parameters (spectral type, $T_{\mathrm{eff}}, R, M$, semimajor axis $a$, and $L_{\mathrm{X}}$ ) in Table 4 were taken from the literature (however, see the notes below the table). We typically used the highest $L_{X}$ in the case of variable X-ray sources. The mass-loss rate was derived using Eq. (1) of Krtička \& Kubát (2012) from the stellar luminosity $L$ and the luminosity class.

We included the parameters of HMXBs in the $L_{X}-t_{X}$ plots in Fig. 1. We assumed that the X-ray source distance $D$ is equal to the semimajor axis $a$. The positions of all HMXBs in the diagrams of $L_{X}$ vs. $t_{X}$ lie outside the forbidden area. Moreover, many HMXBs appear just at the border between the forbidden area and the diagonal strip with a strong influence of X-rays on the wind. This indicates that the winds of these stars are in a selfregulated state. In the self-regulated state an increase in X-ray luminosity causes wind inhibition and therefore a decrease in $L_{\mathrm{X}}$ (Krtička et al. 2012), whereas with a lower X-ray luminosity the influence of X-rays on the wind becomes weaker and the wind is faster, which leads to a stronger X-ray production. The combination of the two effects may keep the location of the star in the diagram of $L_{\mathrm{X}}$ vs. $t_{\mathrm{X}}$ in the vicinity of the border line with forbidden solutions.

Some objects (e.g. IGR J16479-4514, IGR J17252-3616, and CPD-63 ${ }^{\circ} 2495$ ) show strong X-ray variability, which would appear as a vertical shift of their position in the $L_{X}-t_{X}$ plots. A horizontal shift in $L_{X}-t_{X}$ plots is introduced in eccentric systems as a result of the orbital motion (e.g. IGR J11215-5952, CPD$\left.63^{\circ} 2495\right)$. This shows that ionization conditions in the wind may change with time.

Parameters of most HMXBs lie in the area with a strong influence of X-rays on the wind structure (see Fig. 1), leading to the decrease in wind terminal velocity with respect to nonirradiated winds. Within the classical Bondi-Hoyle-Lyttleton picture (Hoyle \& Lyttleton 1941; Bondi \& Hoyle 1944) this affects the accretion rate and the X-ray luminosity. This effect can, for example, explain, why the wind velocity required to explain the X-ray luminosity of $4 \mathrm{U} 2206+54$ is significantly lower than the terminal velocity expected for this type of star (Ribó et al. 2006).

The existence of the forbidden area in the diagram of $L_{\mathrm{X}}$ vs. $t_{\mathrm{X}}$ may be one of the reasons, why the X-ray luminosity of IGR J16479-4514 is by two orders of magnitude lower than that estimated from the Bondi-Hoyle accretion theory (Sidoli et al. 2013). This star with $L_{\mathrm{X}}=1.09 \times 10^{34} \mathrm{erg} \mathrm{s}^{-1}$ and $t_{\mathrm{X}}=0.4$ 
J. Krtička et al.: The effect of the X-ray irradiation on the wind in binaries with hot component
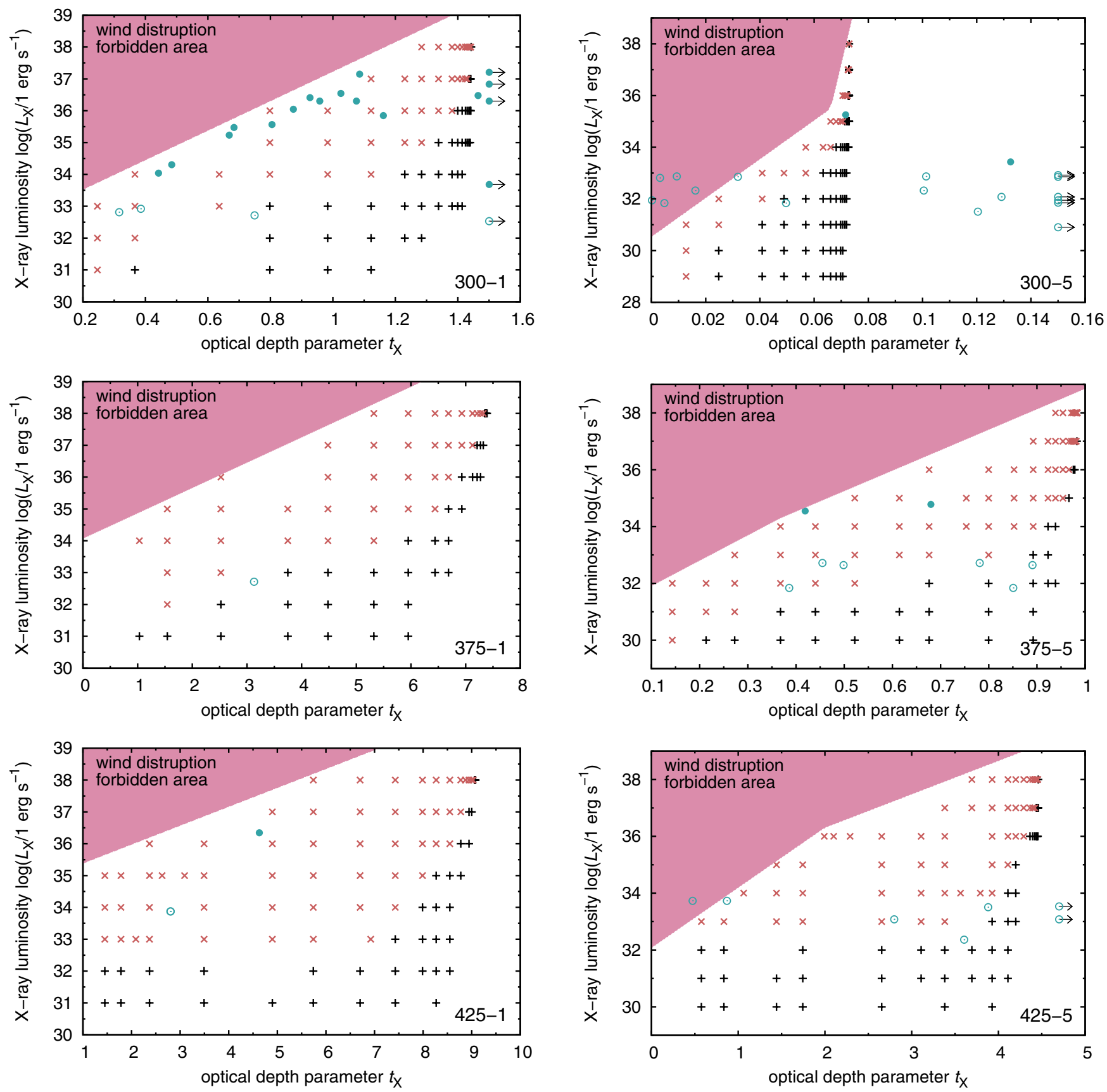

Fig. 1. Regions with different effect of the X-ray irradiation in the diagrams of X-ray luminosity $\left(L_{X}\right)$ vs. the optical depth parameter $\left(t_{X}\right)$. Graphs are plotted for individual model stars in Table 1. Individual symbols denote positions of: models with negligible influence of X-ray irradiation (black plus, +), models where X-ray irradiation leads to the decrease of the wind terminal velocity (red cross, $\times$ ), non-degenerate components of HMXBs from Table 4 (filled circles, ๑), and individual components of X-ray binaries from Table 5 (empty circles, $\odot$ ). The regions of the $L_{X}-t_{X}$ parameters that lead to the wind inhibition are denoted using the shaded area ( $\square$.

lies very close to the forbidden area. Moreover, during flares it reaches peak luminosities of $L_{\mathrm{X}}=6.4 \times 10^{34} \mathrm{erg} \mathrm{s}^{-1}$, which corresponds to the boundary of the forbidden area.

Stars exhibiting Roche-lobe overflow may appear in the forbidden area, since forces other than the radiative force drives the flow. This is likely the case of Cen X-3, which for parameters from Suchy et al. (2008) and Atoyan et al. (2002) gives $t_{X}=0.3$; this is too low for its X-ray luminosity $L_{X}=5.4 \times 10^{37} \mathrm{erg} \mathrm{s}^{-1}$ (Suchy et al. 2008). There are also observational indications of the Roche-lobe overflow in this system (Suchy et al. 2008; Naik et al. 2011).

We also tested additional HMXBs (not listed in Table 4) with $T_{\text {eff }}<25000 \mathrm{~K}$. Their wind parameters may be influenced by the presence of the bistability jump, and consequently, we applied the mass-loss rate predictions of Vink et al. (2000) for these stars. For IGR J00370+6122, with parameters from González-Galán et al. (2014), we derive $t_{\mathrm{X}}=3.3$, which is for $L_{X}=2.5 \times 10^{35} \mathrm{erg} \mathrm{s}^{-1}$ well below the forbidden region in Fig. 1. A similar result was derived for Swift J1700.8-4139. With parameters from Chakrabarty et al. (2002) and Mason et al. (2012) we derive the optical depth parameter $t_{X}=1.5$, which is for $L_{X}=3 \times 10^{36} \mathrm{erg} \mathrm{s}^{-1}$ again well below the threshold in Fig. 1. The object IGR J16318-4848, with $L_{X}=1.3 \times 10^{35} \mathrm{erg} \mathrm{s}^{-1}$ (Filliatre \& Chaty 2004) and $t_{\mathrm{X}}=0.5$ (with parameters from Chaty \& Rahoui 2012), lies just at the border of the forbidden area. 


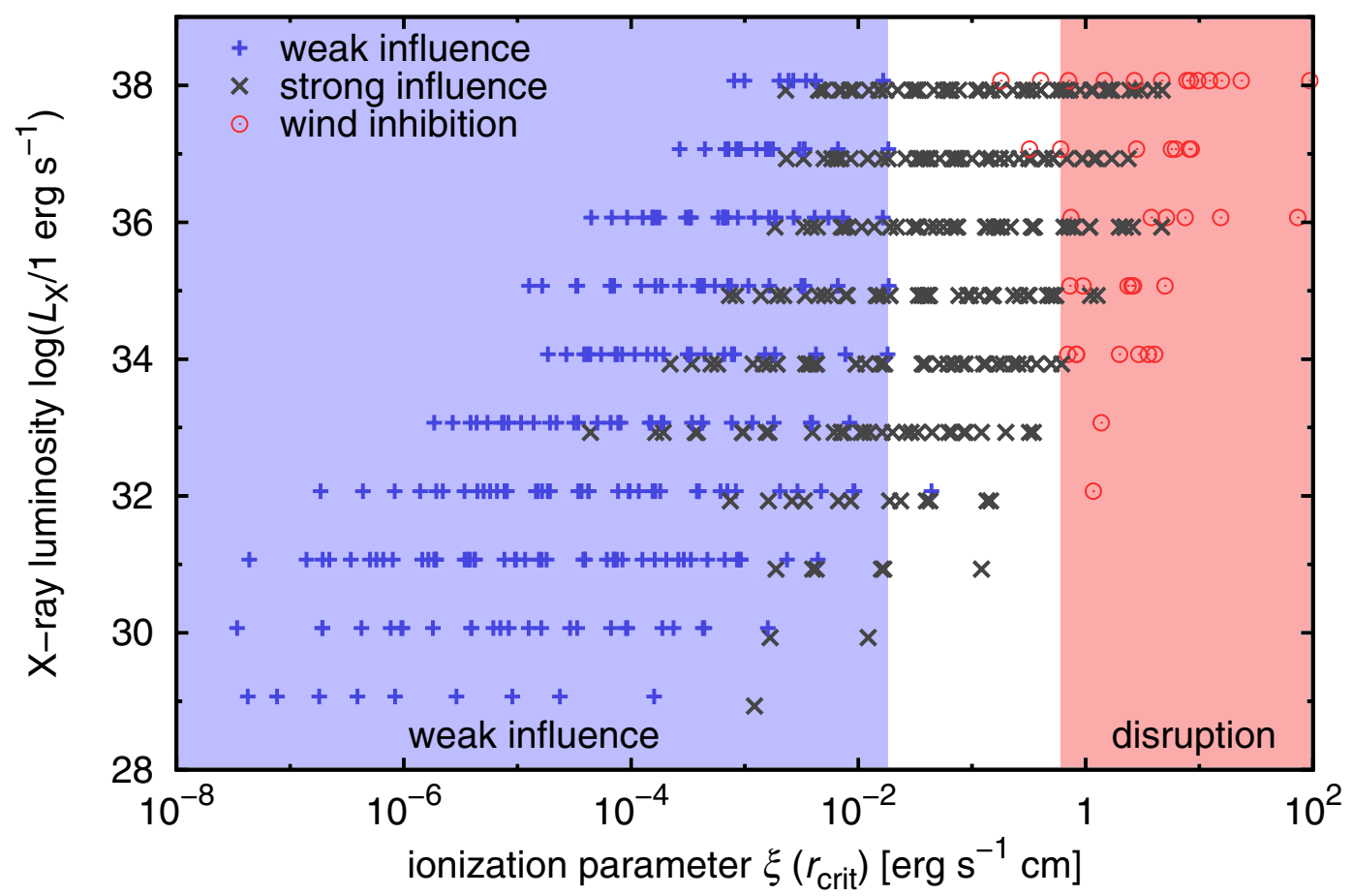

Fig. 2. Parameters of all models from Fig. 1 plotted in the diagram of X-ray luminosity vs. the ionization parameter. The ionization parameter Eq. (5) was evaluated at the wind critical point with radius $r_{\text {crit. }}$ The influence of X-rays is denoted using different symbols. Blue plus signs + denote the weak influence of X-rays (possibly just on the ionization equilibrium), grey crosses $\times$ denote the strong influence of X-rays leading to the decrease of the terminal velocity, and empty red circles $\odot$ denote models with wind inhibition. The individual symbols are slightly vertically shifted for a better readability. The extension of individual regions is also denoted using coloured areas.

\subsection{Binaries with non-degenerate components}

The comparison with observations in the case of massive X-ray binaries with non-degenerate components is complicated by the fact that the X-ray source distance can not be uniquely determined from observations. To overcome this, we assume that the wind collisional front is in equilibrium, which means that the momenta deposited per unit of time and surface by the wind of both components are equal (e.g. Antokhin et al. 2004),

$\rho_{1} v_{1}^{2}=\rho_{2} v_{2}^{2}$,

where the subscripts 1 and 2 denote the wind parameters of the primary and secondary, respectively. Here we study the wind at the intersection of both components. Denoting the radial distances from the individual star centres $D_{1}$ and $D_{2}$ and using the continuity equation, we find

$\frac{D_{1}^{2}}{D_{2}^{2}}=\frac{\dot{M}_{1} v_{1, \infty}}{\dot{M}_{2} v_{2, \infty}}$,

with $a=D_{1}+D_{2}$. Here we assumed that both winds reached their terminal velocities.

Stellar and wind parameters of individual binaries are given in Table 5. The list is based on the known binaries included in the Berghöfer et al. (1997) sample supplemented by several binaries found in the literature. Stellar and binary parameters are taken from the literature. The mass-loss rates were derived using Eq. (1) of Krtička (2014, corrected for actual radius) for mainsequence B stars and Eq. (1) of Krtička \& Kubát (2012) for other stars. For the terminal velocity we assumed the relation between the escape velocity $v_{\text {esc }}$ and the terminal velocity $v_{\infty}=2.6 v_{\text {esc }}$. This relation follows from both observations and theory (Lamers et al. 1995; Krtička \& Kubát 2004).
We included the parameters of X-ray binaries in the $L_{X}-t_{X}$ plots in Fig. 1. If the X-ray emission originates from the windwind collision, then any of the winds should not be inhibited by X-ray emission. Therefore, each binary appears in Fig. 1 and Table 5 twice, once for the primary and once for the secondary.

Most of the binary member parameters studied lie in the allowed part of the $L_{X}-t_{X}$ plots in Fig. 1. This shows that the winds of both components are not inhibited by X-ray emission and therefore $\mathrm{X}$-ray emission in these objects may originate in wind-wind collisions. Many parameters correspond to the region with a strong influence of X-rays on the wind velocity structure. These binaries may be in a self-regulated state, but of a different type than we identified in the HMXBs. In binaries with non-degenerate components, an increase in X-ray luminosity $L_{X}$ causes a decrease in the wind velocity and therefore a decrease in $L_{X}$ (Parkin \& Sim 2013). Similarly, the decrease in $L_{X}$ leads to increase in the terminal velocity, which subsequently causes an increase in $L_{X}$. This may be one of the effects that contribute to the observational conclusion that most binaries are not stronger X-ray sources than corresponding single stars (e.g. Sana et al. 2006b; Antokhin et al. 2008).

Some of the secondaries' parameters lie in the forbidden region in Fig. 1 (these are denoted by a superscript $b$ in Table 5). This indicates that their wind may be inhibited by the X-ray emission. The emission may originate in this case from the collision of the primary wind with the secondary star. It is also possible that 1D models are not applicable for modelling the wind in these systems, or that some parameters are not correct (e.g. mass-loss rate). Another possibility is that the basic stellar parameters (luminosities, masses, and radii) need revision.

For some secondaries, the X-ray source distance $D_{2}$ calculated from Eq. (10) is lower than their radius $R_{2}$ (see a superscript $a$ in Table 5). For these stars, the primary wind may reach 
Table 4. Parameters of HMXBs with neutron star or black hole companion.

\begin{tabular}{|c|c|c|c|c|c|c|c|c|c|c|}
\hline Binary & Sp. Type & $\log \left(L / L_{\odot}\right)$ & $T_{\text {eff }}[\mathrm{K}]$ & $R\left[R_{\odot}\right]$ & $M\left[M_{\odot}\right]$ & $a\left[R_{\odot}\right]$ & $L_{\mathrm{X}}\left[\mathrm{erg} \mathrm{s}^{-1}\right]$ & $\dot{M}\left[M_{\odot} \mathrm{yr}^{-1}\right]$ & $t_{\mathrm{X}}$ & Reference \\
\hline $2 \mathrm{~S} 0114+650^{f}$ & B1Iae & 5.61 & 24000 & 37 & 16 & 54 & $1.1 \times 10^{36}$ & $6.8 \times 10^{-7}$ & 0.9 & $1,2,37$ \\
\hline Vela X-1 ${ }^{f}$ & B0Ia & 5.63 & 27000 & 30 & 23.5 & 53.4 & $3.5 \times 10^{36}$ & $7.5 \times 10^{-7}$ & 1.0 & $3,4,5$ \\
\hline $4 \mathrm{U} 1700-377^{f}$ & O6.5Iaf & 6.02 & 40200 & 21.2 & 58 & 37 & $2.2 \times 10^{36}$ & $4.6 \times 10^{-6}$ & 4.6 & $6,7,8$ \\
\hline IGR J16418-4532 & O8.5I & 5.69 & 32800 & 21.7 & 30 & 31.6 & $2 \times 10^{36}$ & $9.8 \times 10^{-7}$ & 1.0 & 9,38 \\
\hline IGR J18029-2016 & B1I & 5.59 & 32500 & 19.8 & 20.2 & 33.1 & $2 \times 10^{36}$ & $6.3 \times 10^{-7}$ & 1.1 & 10,29 \\
\hline IGR J16479-4514 & O8.5Iab & 5.52 & $31000^{a}$ & 20 & 35 & 31.6 & $1.09 \times 10^{34}$ & $4.5 \times 10^{-7}$ & 0.4 & 11 \\
\hline IGR J17252-3616 & B0Ia & 5.79 & 30000 & 29 & 15 & 51 & $1.6 \times 10^{37}$ & $1.5 \times 10^{-6}$ & 11.3 & $12,13,40$ \\
\hline IGR J18483-0311 & B0.5Ia & 5.57 & 24600 & 33.8 & 33 & 95.7 & $3.7 \times 10^{35}$ & $5.7 \times 10^{-7}$ & 0.8 & $14,15,42$ \\
\hline IGR J18450-0435 & O9Ia & 5. & 30000 & 23 & 30 & 72 & $7 \times 10^{35}$ & $6.0 \times 10^{-7}$ & 1.2 & $16,17,43$ \\
\hline $\mathrm{X} \operatorname{Per}^{f}$ & Bove & 4.69 & $32000^{a}$ & $7.2^{a}$ & 15.5 & 420 & $2.7 \times 10^{33}$ & $2.2 \times 10^{-8}$ & 0.1 & 18,30 \\
\hline IGR J11215-5952 & B0.5Ia & 5.73 & 24700 & 40 & 29 & $80^{b}$ & $3 \times 10^{36}$ & $1.2 \times 10^{-6}$ & 1.5 & 19,20 \\
\hline PSR B1259-63 $3^{f, c}$ & $09.5 \mathrm{Ve}$ & 4.9 & $34000^{d}$ & $8.1^{d}$ & 10 & 1100 & $3.5 \times 10^{34}$ & $5.5 \times 10^{-8}$ & 0.4 & 21,22 \\
\hline IGR J19140+0951 & B0.5I & 5.47 & $28000^{a}$ & $23.2^{a}$ & $25.4^{a}$ & 62.5 & $3 \times 10^{35}$ & $3.6 \times 10^{-7}$ & 0.7 & 23 \\
\hline $4 U 2206+54$ & $09.5 \mathrm{Ve}$ & 4.59 & 30000 & 7.3 & 16 & 53 & $1.8 \times 10^{35}$ & $1.4 \times 10^{-8}$ & 0.1 & 24 \\
\hline 4 U $1907+09$ & O8.5Iab & 5.6 & 29760 & 26.2 & 26.0 & 54 & $2 \times 10^{36}$ & $9.6 \times 10^{-7}$ & 1.6 & 25 \\
\hline LS $5039^{f}$ & O6.5V & 5.19 & 37500 & 9.3 & 22.9 & 34.5 & $6 \times 10^{34}$ & $2.0 \times 10^{-7}$ & 0.7 & $26,39,44,45$ \\
\hline Cyg X-1 ${ }^{f}$ & O9.7Iab & 5.57 & 32000 & $19.9^{e}$ & 24.0 & 42.4 & $1.4 \times 10^{37}$ & $5.7 \times 10^{-7}$ & 1.1 & $27,28,41$ \\
\hline IGR J16465-4507 & 09.5Ia & 5.69 & $30000^{a}$ & 26 & 27.8 & 124 & $6.8 \times 10^{36}$ & $9.9 \times 10^{-7}$ & 2.4 & 31,32 \\
\hline IGR J17544-2619 & $\mathrm{O} 9 \mathrm{Ib}$ & 5.53 & 31000 & 20.3 & 26.5 & 36.3 & $1.7 \times 10^{35}$ & $4.7 \times 10^{-7}$ & 0.7 & 33,34 \\
\hline IGR J16207-5129 & B1Ia & 5.4 & $29000^{a}$ & 20 & 20 & 40 & $2 \times 10^{34}$ & $2.6 \times 10^{-7}$ & 0.5 & 35,36 \\
\hline XTE X1739-302 & O8.5Iab & 5.71 & $33000^{a}$ & $21.9^{a}$ & $30.8^{a}$ & 173 & $4.8 \times 10^{33}$ & $1.1 \times 10^{-6}$ & 3.0 & 46,47 \\
\hline IGR J17354-3255 & O9.5Iab & 5.56 & $30000^{a}$ & $22.4^{a}$ & $28.8^{a}$ & 54 & $2.6 \times 10^{36}$ & $5.4 \times 10^{-7}$ & 0.9 & 48,49 \\
\hline
\end{tabular}

Notes. Stellar parameters are taken from literature except for the mass-loss rate, for which we used fits of Krtička \& Kubát (2012). ${ }^{(a)}$ Derived from the spectral type using the expressions of Martins et al. (2005a). ${ }^{(b)}$ Periastron distance. ${ }^{\left({ }^{c}\right)}$ Possible disk accretion. ${ }^{(d)}$ Polar values. ${ }^{(e)}$ In the direction to the companion. ${ }^{(f)}$ Some alternative designations: 2S 0114+650 (V662 Cas), Vela X-1 (GP Vel, HD 77581), 4U 1700-377 (V884 Sco, HD 153919), X Per (HR 1209), PSR B1259-63 (CPD-632495), LS 5039 (V479 Sct), and Cyg X-1 (V1357 Cyg, HD 226868).

References. (1) Reig et al. (1996); (2) Farrell et al. (2008); (3) van Kerkwijk et al. (1995); (4) Straižys \& Kuriliene (1981); (5) Watanabe et al. (2006); (6) Coleiro \& Chaty (2013); (7) Clark et al. (2002); (8) van der Meer et al. (2005); (9) Drave et al. (2013); (10) Mason et al. (2011a); (11) Sidoli et al. (2013); (12) Mason et al. (2011b); (13) Manousakis et al. (2012); (14) Romano et al. (2010); (15) Rahoui \& Chaty (2008); (16) Goossens et al. (2013); (17) Sguera et al. (2007); (18) Tomsick \& Muterspaugh (2010); (19) Romano et al. (2007); (20) Lorenzo et al. (2014); (21) Sushch et al. (2013); (22) Sierpowska-Bartosik \& Bednarek (2008); (23) Prat et al. (2008); (24) Ribó et al. (2006); (25) Cox et al. (2005); (26) Casares et al. (2005); (27) Herrero et al. (1995); (28) Hadrava \& Čechura (2012); (29) Hill et al. (2005); (30) Berghöfer et al. (1997); (31) Clark et al. (2010); (32) La Parola et al. (2010); (33) Drave et al. (2014); (34) Pellizza et al. (2006); (35) Tomsick et al. (2009); (36) Bodaghee et al. (2010); (37) Hall et al. (2000); (38) Chaty et al. (2008); (39) Herrero et al. (2002); (40) Thompson et al. (2007); (41) Yan et al. (2008); (42) Searle et al. (2008); (43) Zurita Heras \& Walter (2009); (44) Moldón et al. (2012); (45) McSwain et al. (2004); (46) Drave et al. (2010); (47) Bodaghee et al. (2011); (48) Sguera et al. (2011); (49) Sguera (2013).

the secondary and the secondary wind may be completely inhibited in the direction toward the primary. Parameters of these secondaries are not plotted in Fig. 1. Our models may also be oversimplified in this case. For example, we neglect the radiative force from the secondary acting on the primary wind (Gayley et al. 1997), which may avert the secondary wind inhibition. Moreover, a significant part of X-rays may also originate in shocks in supersonic winds similar to the case of single stars (Lucy \& White 1980; Owocki et al. 1988; Feldmeier et al. 1997; Babel \& Montmerle 1997; ud-Doula et al. 2014).

\section{Implications for other massive X-ray sources}

\subsection{Be/X-ray binaries}

The X-ray emission in Be/X-ray binaries originates from the accretion of the Be star disk material on the compact source (see Reig 2011, for a review). The origin of the disk is still a matter of debate. Some theories propose that the disk is fed by the wind. We used the list of Be/X-ray binaries given in Krtička et al. (2015) to test whether the radiatively driven wind can survive the strong X-rays source in $\mathrm{Be} / \mathrm{X}$-ray binaries. In many Be/X-ray binaries the compact source is located at large distance from the Be star, and, consequently, it can not disrupt its wind. However, in some $\mathrm{Be} / \mathrm{X}$-ray binaries the compact object is located in such proximity to the Be star, that it disrupts its wind. This is another argument that disfavours the wind origin of Be star disks.

\subsection{X-ray binaries in the Magellanic Clouds}

X-ray binaries in the Magellanic Clouds typically show large $\mathrm{X}$-ray luminosities of the order of $10^{37}-10^{38} \mathrm{erg} \mathrm{s}^{-1}$. Because of lower metallicity of LMC and SMC compared to our Galaxy, the wind mass-loss rates are expected to be also correspondingly lower (e.g. Bouret et al. 2003). We tested three LMC and SMC binaries, LMC X-1, LMC X-4, and SMC X-1, with parameters taken from literature (Orosz et al. 2009; Hung et al. 2010; Rawls et al. 2011; Li \& Li 2014). In all these cases, the corresponding stellar parameters lie in a forbidden zone, indicating that the wind of these stars is inhibited in the direction toward the companion and the X-rays most likely originate in the Roche lobe overflow. There is an observational support for this indicating that many of these binaries indeed likely fill their Roche lobes (Negueruela \& Coe 2002; Val Baker et al. 2005; Orosz et al. 2009).

\subsection{X-rays from wind shocks}

The X-ray emission of single non-magnetic hot stars presumably originates in the shocks caused by the instability connected 
Table 5. Parameters of binaries with non-degenerate components.

\begin{tabular}{|c|c|c|c|c|c|c|c|c|c|c|c|}
\hline Binary & Sp. Type & $\log \left(L / L_{\odot}\right)$ & $\begin{array}{l}T_{\text {eff }} \\
{[\mathrm{K}]}\end{array}$ & $\begin{array}{c}R \\
{\left[R_{\odot}\right]}\end{array}$ & $\begin{array}{c}M \\
{\left[M_{\odot}\right]}\end{array}$ & $\begin{array}{c}\dot{M} \\
{\left[M_{\odot} \mathrm{yr}^{-1}\right]}\end{array}$ & $\begin{array}{c}v_{\infty} \\
{\left[\mathrm{km} \mathrm{s}^{-1}\right]}\end{array}$ & $t_{\mathrm{X}}$ & $\begin{array}{c}a \\
{\left[R_{\odot}\right]}\end{array}$ & $\begin{array}{c}L_{\mathrm{X}} \\
{\left[\mathrm{erg} \mathrm{s}^{-1}\right]}\end{array}$ & Reference \\
\hline \multirow[t]{2}{*}{ HR 8281} & $06.5 \mathrm{~V}$ & 5.7 & 41200 & 13.9 & 40.2 & $2.0 \times 10^{-6}$ & 2290 & 3.6 & 40 & $2.3 \times 10^{32}$ & $1,2,3$ \\
\hline & $\mathrm{O}^{9} \mathrm{~V}^{a}$ & 5.02 & 34900 & 8.9 & 22.4 & $9.7 \times 10^{-8}$ & 2400 & & & & \\
\hline \multirow[t]{2}{*}{ HD 152219} & O9.5III & 5.07 & 31900 & 11.2 & 26.2 & $8.0 \times 10^{-8}$ & 2320 & 0.2 & 37.1 & $8 \times 10^{30}$ & 4 \\
\hline & $\mathrm{B} 1 \mathrm{~V}^{a}$ & 3.74 & 21800 & 5.2 & 10.3 & $3.4 \times 10^{-10}$ & 2250 & & & & \\
\hline \multirow[t]{2}{*}{ HD 47129} & O8I & 5.35 & 33500 & 14.1 & 54. & $2.0 \times 10^{-7}$ & 2980 & 0.4 & 128.5 & $8.34 \times 10^{32}$ & 5,30 \\
\hline & O7.5III & 5.09 & 33000 & 10.8 & 56. & $9.0 \times 10^{-8}$ & 3560 & 0.2 & & & \\
\hline \multirow[t]{2}{*}{ HR 6187} & O3V & 5.78 & 44500 & 13.14 & 63 & $2.9 \times 10^{-6}$ & 3090 & 3.9 & 38.0 & $3.2 \times 10^{33}$ & $1,6,7,8$ \\
\hline & $05.5 \mathrm{~V}^{a}$ & 5.28 & 39000 & 9.54 & 40. & $3.0 \times 10^{-7}$ & 3100 & & & & \\
\hline \multirow[t]{2}{*}{ HR 6736} & $03.5 \mathrm{~V}$ & 5.75 & 43850 & $13.1^{c}$ & 55 & $2.6 \times 10^{-6}$ & 2860 & 6.9 & 4100 & $1.2 \times 10^{33}$ & 1,9 \\
\hline & $\mathrm{O} 5 \mathrm{~V}$ & 5.5 & 40850 & $11.2^{c}$ & 36 & $8.1 \times 10^{-7}$ & 2560 & 2.8 & & & \\
\hline \multirow[t]{2}{*}{ V712 Car } & O3I & 6.06 & 43000 & 19.3 & 82.7 & $5.4 \times 10^{-6}$ & 2720 & 2.8 & 53 & $7.5 \times 10^{33}$ & 10,11 \\
\hline & O3I & 6.06 & 43000 & 19.3 & 81.9 & $5.4 \times 10^{-6}$ & 2700 & 2.8 & & & \\
\hline \multirow[t]{2}{*}{ HR 65} & O9III & 5.2 & $32000^{c}$ & 13 & 15 & $1.5 \times 10^{-7}$ & 1490 & 0.3 & 32 & $7.2 \times 10^{32}$ & 12,13 \\
\hline & O9III & 4.83 & $32000^{c}$ & 8.5 & 21 & $2.7 \times 10^{-8}$ & 2430 & 0.0 & & & \\
\hline \multirow[t]{2}{*}{ HR 5664} & O7V & 5.27 & 37500 & 10.2 & 21.6 & $2.9 \times 10^{-7}$ & 2090 & 0.9 & 33.8 & $6.9 \times 10^{31}$ & 1,14 \\
\hline & $09.5 \mathrm{~V}^{b}$ & 4.64 & 33000 & 6.4 & 12.4 & $1.7 \times 10^{-8}$ & 2140 & 0.0 & & & \\
\hline \multirow[t]{2}{*}{ HR 8406} & O8.5III & 5.11 & 32000 & 11.7 & 16.9 & $9.7 \times 10^{-8}$ & 1750 & 0.2 & 25.5 & $7.1 \times 10^{31}$ & 1,15 \\
\hline & $09.5 \mathrm{~V}^{a}$ & 4.69 & 28000 & 9.4 & 6.7 & $2.2 \times 10^{-8}$ & 1230 & & & & \\
\hline \multirow[t]{2}{*}{ HD 93206A } & O9.7I & 5.7 & 32000 & 23 & 40 & $1.0 \times 10^{-6}$ & 1780 & 2.0 & 116 & $3.4 \times 10^{32}$ & 16,29 \\
\hline & $\mathrm{B} 2 \mathrm{~V}^{a}$ & 3.7 & 20000 & 5.9 & 10 & $2.3 \times 10^{-10}$ & 2080 & & & & \\
\hline \multirow[t]{2}{*}{ HD 93206B } & O8III & 5.3 & 32600 & 14 & 14 & $2.3 \times 10^{-7}$ & 1310 & 0.6 & 49 & $1.2 \times 10^{32}$ & 16,29 \\
\hline & O9V & 4.9 & 32500 & 8.9 & 28 & $5.6 \times 10^{-8}$ & 2750 & 0.1 & & & \\
\hline \multirow[t]{2}{*}{ V1007 Sco } & O7.5III & 5.4 & 34000 & 14.47 & 27.24 & $3.7 \times 10^{-7}$ & 1950 & 0.5 & 51.7 & $4.4 \times 10^{32}$ & 17,18 \\
\hline & O7III & 5.5 & 34350 & 15.99 & 27.88 & $5.9 \times 10^{-7}$ & 1810 & 0.9 & & & \\
\hline \multirow[t]{2}{*}{ HR 1931} & $09.5 \mathrm{~V}$ & 4.64 & 32600 & $6.6^{c}$ & 19 & $1.8 \times 10^{-8}$ & 2650 & 0.1 & 375 & $2.1 \times 10^{32}$ & $1,19,31$ \\
\hline & $\mathrm{B} 0 \mathrm{~V}^{b}$ & 4.45 & $30600^{c}$ & $6.0^{c}$ & 15.4 & $2.6 \times 10^{-9}$ & 2520 & 0.0 & & & \\
\hline \multirow[t]{2}{*}{ HR 6535} & O6V & 5.26 & $39000^{c}$ & 9.4 & 32 & $2.9 \times 10^{-7}$ & 2750 & 0.8 & 56.4 & $5.2 \times 10^{32}$ & $1,20,21,22$ \\
\hline & O7V & 5.17 & $37000^{c}$ & 9.4 & 32 & $1.9 \times 10^{-7}$ & 2790 & 0.5 & & & \\
\hline \multirow[t]{2}{*}{ HR 1899} & O9III & 4.81 & $32000^{c}$ & 8.3 & 23 & $2.5 \times 10^{-8}$ & 2580 & 0.1 & 80 & $7.4 \times 10^{32}$ & $1,21,23,24$ \\
\hline & $\mathrm{B} 0.5 \mathrm{~V}^{b}$ & 4.19 & $28000^{c}$ & 5.3 & 13 & $1.8 \times 10^{-9}$ & 2480 & 0.0 & & & \\
\hline \multirow[t]{2}{*}{ HR 1852} & O9.5II & 5.15 & $31000^{c}$ & 13 & 11.2 & $7.8 \times 10^{-8}$ & 1250 & 0.3 & 44 & $6.5 \times 10^{32}$ & $1,21,25,26$ \\
\hline & $\mathrm{B} 0.5 \mathrm{III}^{b}$ & 4.2 & $29000^{c}$ & 5 & 5.6 & $1.5 \times 10^{-9}$ & 1640 & 0.0 & & & \\
\hline \multirow[t]{2}{*}{ V918 Sco } & O7.5I & 5.9 & 35100 & 24.3 & 57 & $2.7 \times 10^{-6}$ & 2000 & 3.1 & 91 & $5.2 \times 10^{32}$ & 1,27 \\
\hline & O9.7I & 5.79 & 30500 & 28.1 & 37 & $1.5 \times 10^{-6}$ & 1430 & 0.7 & & & \\
\hline \multirow[t]{2}{*}{ HR 7767} & O8.5III & 5.25 & 32500 & $13.4^{c}$ & 23 & $1.9 \times 10^{-7}$ & 1900 & 0.7 & 620 & $8.9 \times 10^{31}$ & 1,28 \\
\hline & $\mathrm{B} 2.5 \mathrm{~V}^{b}$ & 3.29 & 20000 & $3.7^{c}$ & 9 & $3.5 \times 10^{-11}$ & 2500 & 0.0 & & & \\
\hline \multirow[t]{2}{*}{ DH Cep } & $\mathrm{O} 5.5 \mathrm{~V}$ & 5.37 & 44000 & 8.31 & 29.4 & $4.5 \times 10^{-7}$ & 2720 & 0.9 & 26.4 & $5.4 \times 10^{33}$ & 12,32 \\
\hline & $06.5 \mathrm{~V}^{b}$ & 5.27 & 43000 & 7.76 & 25.0 & $2.9 \times 10^{-7}$ & 2620 & 0.5 & & & \\
\hline \multirow[t]{2}{*}{ V1034 Sco } & O9V & 4.78 & 33200 & 7.45 & 16.8 & $3.3 \times 10^{-8}$ & 2310 & 0.1 & 22.65 & $3.2 \times 10^{31}$ & 33,34 \\
\hline & $\mathrm{B} 1 \mathrm{~V}^{a}$ & 3.87 & 26200 & 4.18 & 9.4 & $5.8 \times 10^{-10}$ & 2390 & & & & \\
\hline \multirow[t]{2}{*}{ V1294 Sco } & O9V & 5.1 & 34000 & 10.3 & 24.5 & $1.4 \times 10^{-7}$ & 2320 & 0.4 & 42.8 & $6.9 \times 10^{31}$ & 35 \\
\hline & $09.7 \mathrm{~V}$ & 4.71 & 31200 & 7.8 & 18.2 & $2.4 \times 10^{-8}$ & 2370 & 0.0 & & & \\
\hline HD 93205 & $\mathrm{O} 3 \mathrm{~V}$ & 6.18 & 49000 & $17.2^{c}$ & 45 & $1.8 \times 10^{-5}$ & 1130 & 60.8 & 56.3 & $3.4 \times 10^{33}$ & 36 \\
\hline & $\mathrm{O} 8 \mathrm{~V}^{a}$ & 5.11 & 36500 & $9.0^{c}$ & 20 & $1.4 \times 10^{-7}$ & 2200 & & & & \\
\hline
\end{tabular}

Notes. Stellar parameters are taken from literature except for the mass-loss rate, for which we used fits of Krtička \& Kubát (2012) and Krtička (2014). Alternative designations: HR 8281 (HD 206267), HD 152219 (V1292 Sco), HD 47129 (V640 Mon, HR 2422, and Plaskett star), HR 6187 (HD 150136), HR 6736 (9 Sgr, HD 164794), V712 Car (WR 20a), HR 65 (AO Cas, HD 1337), HR 5664 ( $\delta$ Cir, HD 135240$)$, HR 8406 (14 Cep, LZ Cep, HD 209481), HD 93206 (QZ Car), V1007 Sco (HD 152248), HR 1931 ( $\sigma$ Ori, HD 37468), HR 6535 (V1036 Sco, HD 159176), HR 1899 ( $\iota$ Ori A, HD 37043), HR 1852 ( $\delta$ Ori, HD 36486), V918 Sco (HR 6164, HD 149404), HR 7767 (HD 193322), and HD 93205 (V560 Car). ${ }^{(a)}$ The primary wind reaches the secondary surface $\left(D_{2}<R_{2}\right) .{ }^{(b)}$ The wind parameters lie in the forbidden area. ${ }^{(c)}$ Derived from the effective temperature or spectral type using the expressions of Harmanec (1988) for main-sequence B stars and Martins et al. (2005a) for others.

References. (1) Berghöfer et al. (1997); (2) Burkholder et al. (1997); (3) Stickland (1995); (4) Sana et al. (2006a); (5) Linder et al. (2006); (6) Mahy et al. (2012); (7) Sana et al. (2013); (8) Sanchez-Bermudez et al. (2013); (9) Rauw et al. (2012); (10) Rauw et al. (2005); (11) Montes et al. (2013); (12) Chlebowski et al. (1989); (13) Sahade \& Brandi (1991); (14) Penny et al. (2001); (15) Mahy et al. (2011); (16) Parkin et al. (2011); (17) Sana et al. (2004); (18) Mayer et al. (2008); (19) Simón-Díaz et al. (2011); (20) Pachoulakis (1996); (21) Gies (2003); (22) Chlebowski \& Garmany (1991); (23) Marchenko et al. (2000); (24) Bagnuolo et al. (2001); (25) Harvin et al. (2002); (26) Mayer et al. (2010); (27) Rauw et al. (2001); (28) ten Brummelaar et al. (2011); (29) Leung et al. (1979); (30) Linder et al. (2008); (31) Najarro et al. (2011); (32) Sturm \& Simon (1994); (33) Sana et al. (2005); (34) Bouzid et al. (2005); (35) Sana et al. (2008); (36) Antokhina et al. (2000). 
with line driving (Owocki et al. 1988; Feldmeier et al. 1997). There may be a feedback effect of the shock generated X-rays on the ionization structure of the wind. The typical X-ray luminosity scales with the stellar luminosity, $L_{\mathrm{X}} \approx 10^{-7} L$ (Sana et al. 2006b; Antokhin et al. 2008), however, simulations predict stochastic X-ray variability on some level. Taking as an example the predicted $L_{\mathrm{X}}$ variations of the $09.7 \mathrm{Ib}$ star $\zeta$ Ori A (in the order of $10^{33}-10^{34} \mathrm{erg} \mathrm{s}^{-1}$ ) from the numerical simulations of Feldmeier et al. (1997) and assuming the distance of X-ray source from the star $1.5 R_{*}-5 R_{*}$, the derived value of the $t_{\mathrm{X}}$ parameter is $t_{\mathrm{X}} \approx 0.5-1.5$. This lies in the region of a strong influence of X-rays on the wind ionization state (see Fig. 1 for a model 300-1). Consequently, shock generated X-rays may cause kinks in the velocity profile, which in turn may lead to moving absorption features in the P Cygni line profiles (e.g. Cranmer \& Owocki 1996) or increasing wind inhomogeneities.

For some low-luminosity $\mathrm{O}$ stars the predicted mass-loss rates are significantly higher than those inferred from observations (e.g. Bouret et al. 2003; Martins et al. 2005b). This disagreement is termed as a "weak wind problem". This problem could possibly be explained as a consequence of X-ray influence on the mass-loss rate (Drew et al. 1994). For a typical luminosity of stars showing the weak wind problem, which have $\log \left(L / L_{\odot}\right)=5$, the X-ray luminosity can be inferred using the relation $L_{X} \approx 10^{-7} L$ (Sana et al. 2006b; Antokhin et al. 2008) as $\log \left(L_{\mathrm{X}} / 1 \mathrm{erg} \mathrm{s}^{-1}\right) \approx 31.6$. If an effect of X-rays on the wind is to appear for these low X-ray luminosities, the X-ray source has to be in a very close proximity to the $\operatorname{star}\left(D \lesssim 1.5 R_{*}\right.$, see Fig. 4). However, X-rays typically originate at larger distances (Feldmeier et al. 1997). Moreover, in our calculations we assumed one spatially localized X-ray source, which is unlikely if the X-rays have to influence the entire wind. For a spatially distributed X-ray source the effect of X-ray ionization is expected to be lower. Consequently, our results disfavour this explanation of "weak wind problem", and the weak wind problem seems to be more likely caused by an excessively large cooling length in the post-shock region (Lucy \& White 1980; Martins et al. 2005b; Cohen et al. 2008; Krtička \& Kubát 2009; Lucy 2012).

\subsection{Winds in active galactic nuclei}

The X-ray overionization may also be problematic for linedriven winds in the active galactic nuclei. Using typical parameters of these winds in simulations of Higginbottom et al. (2014) $\left(L_{\mathrm{X}}=10^{44} \mathrm{erg} \mathrm{s}^{-1}\right.$ and $\left.D=10^{16} \mathrm{~cm}\right)$, we derive the ionization parameter $\xi$ of the order of unity (neglecting absorption) even for the densest regions with $\rho=10^{-12} \mathrm{~g} \mathrm{~cm}^{-3}$. Since typical wind densities are by two to four orders of magnitude lower, this supports the conclusion that the winds of active galactic nuclei are strongly inhibited, unless a strong absorption source is present (Higginbottom et al. 2014).

\section{Discussion and conclusions}

We study the effect of external X-ray irradiation on hot star winds in massive binaries. Hot star winds are driven by light absorption in the lines of heavier elements, therefore the wind acceleration is sensitive to the ionization stage of the wind. We used our own computer code to calculate NLTE wind models for typical O star and X-ray source parameters found in massive binaries to estimate the influence of external X-ray irradiation.

The influence of X-rays is determined by the X-ray luminosity, and the $\mathrm{X}$-ray optical depth between a given point in the wind and the X-ray source, which follows from the distance to the X-ray source and X-ray opacity along the path. Therefore, our results can be interpreted in the diagrams of X-ray luminosity vs. the optical depth parameter (in this paper referred to as the diagrams $L_{\mathrm{X}}$ vs. $t_{\mathrm{X}}$ ). The effects of X-rays are negligible in binaries with low X-ray luminosities or large distances between the binary component with wind and the X-ray source. In this kind of a case the external irradiation leads just to a modification of the wind ionization state, which has a relatively low influence on the radiative driving.

With increasing X-ray luminosity, the influence of X-rays on the wind structure becomes stronger. There appears to be a typical kink in the velocity profile at the point where the X-ray overionization is so strong that it lowers the radiative force so much that is not able to accelerate the wind any more. With decreasing distance between the star and the X-ray source the kink moves towards the star. If the kink approaches the critical point, where the mass-loss rates is determined, the wind becomes inhibited by X-rays. Consequently, there is a forbidden area in the diagram of $L_{X}$ vs. $t_{X}$ with high X-ray luminosities and low optical depth parameters, where the X-ray ionization leads to wind inhibition.

We compared the positions of known wind-accreting, highmass X-ray binaries in the diagrams of X-ray luminosity vs. the optical depth parameter with respect to the location of the forbidden area. All primaries of binaries with compact components lie outside the forbidden area, which is consistent with our wind models. Many of primaries lie close to the border of the forbidden area, which means that the increase in X-ray luminosity would lead to wind inhibition. This indicates that the X-ray luminosities of HMXBs are self-regulated.

The X-rays may also strongly affect the ionization state of circumstellar matter in colliding wind binaries. For many of them we predict a strong influence of external irradiation on the wind ionization state, pointing to wind self-regulation of another kind. In some cases the wind of secondary may be inhibited by the X-ray irradiation. We also discussed the application of our results for the X-rays generated by wind instabilities and for disk winds in active galactic nuclei.

There are further observational tests of our models. The decrease in wind terminal velocity may be detectable for individual stars as the shift of the blue edge of the UV P Cygni line profiles (Kaper et al. 1993) or as the shift of the X-ray emission lines (Watanabe et al. 2006). Moreover, the kink in the velocity profile found in the models with strong X-ray ionization may lead to a localized stronger absorption in the blue part of the P Cygni line profiles resembling the discrete absorption components (DACs, Kaper et al. 1994; Cranmer \& Owocki 1996).

Despite a good agreement between observations and theory, our models have limitations. First, we assume a spherical symmetry and solve the wind equations only in the direction towards the X-ray source. While this may be applicable to estimate the overall influence of X-ray irradiation, we are missing many important observational effects that are connected with departures of the wind from spherical symmetry (Friend \& Castor 1982; Blondin et al. 1990; Feldmeier et al. 1996). We also neglected the time-dependent phenomena, which may be important especially in binaries with eccentric orbits. Moreover, there is a mounting evidence that hot star winds show small-scale structures (clumping). This may weaken the effect of X-ray ionization because of increased recombination (Oskinova et al. 2012).

Regardless of these limiting assumptions, our models clearly demonstrate the effect of X-rays on the ionization balance of stellar winds in massive binaries. Depending on the strength of 
the X-ray source and its distance from the wind losing-star, the $\mathrm{X}$-rays are able, via their influence on the ionization structure, to change the wind driving force and, consequently, the properties of the wind. In a limiting case of a strong X-ray source, this leads to wind inhibition. This inhibition is supported by observed properties of binaries.

Acknowledgements. This research was supported GA ČR 13-10589S. Access to computing and storage facilities owned by parties and projects contributing to the National Grid Infrastructure MetaCentrum, provided under the programme "Projects of Large Infrastructure for Research, Development, and Innovations" (LM2010005), is greatly appreciated.

\section{References}

Abbott, D. C. 1980, ApJ, 242, 1183

Antokhin, I. I., Owocki, S. P., \& Brown, J. C. 2004, ApJ, 611, 434

Antokhin, I. I., Rauw, G., Vreux, J.-M., van der Hucht, K. A., \& Brown, J. C. 2008, A\&A, 477, 593

Antokhina, E. A., Moffat, A. F. J., Antokhin, I. I., Bertrand, J.-F., \& Lamontagne, R. 2000, ApJ, 529, 463

Atoyan, A. M., Aye, K.-M., Chadwick, P. M., et al. 2002, A\&A, 383, 864

Babel, J., \& Montmerle, T. 1997, A\&A, 323, 121

Bagnuolo, W. G., Jr., Riddle, R. L., Gies, D. R., \& Barry, D. J. 2001, ApJ, 554, 362

Berghöfer, T. W., Schmitt, J. H. M. M., Danner, R., \& Cassinelli, J. P. 1997, A\&A, 322, 167

Blondin, J. M., Kallman, T. R., Fryxell, B. A., \& Taam, R. E. 1990, ApJ, 356, 591

Bodaghee, A., Tomsick, J. A., Rodriguez, J., et al. 2010, ApJ, 719, 451

Bodaghee, A., Tomsick, J. A., Rodriguez, J., et al. 2011, ApJ, 727, 59

Bondi, H., \& Hoyle, F. 1944, MNRAS, 104, 273

Bouret, J.-C., Lanz, T., Hillier, D. J., et al. 2003, ApJ, 595, 1182

Bouzid, M. Y., Sterken, C., \& Pribulla, T. 2005, A\&A, 437, 769

Burkholder, V., Massey, P., \& Morrell, N. 1997, ApJ, 490, 328

Casares, J., Ribó, M., Ribas, I., et al. 2005, MNRAS, 364, 899

Chakrabarty, D., Wang, Z., Juett, A. M., Lee, J. C., \& Roche, P. 2002, ApJ, 573, 789

Chaty, S., \& Rahoui, F. 2012, ApJ, 751, 150

Chaty, S., Rahoui, F., Foellmi, C., et al. 2008, A\&A, 484, 783

Chlebowski, T., \& Garmany, C. D. 1991, ApJ, 368, 241

Chlebowski, T., Harnden, F. R., \& Sciortino, S. 1989, ApJ, 341, 427

Clark, J. S., Goodwin, S. P., Crowther, P. A., et al. 2002, A\&A, 392, 909

Clark, D. J., Sguera, V., Bird, A. J., et al. 2010, MNRAS, 406, L75

Cooke, B. A., Fabian, A. C., \& Pringle, J. E. 1978, Nature, 273, 645

Cohen, D. H., Kuhn, M. A., Gagné, M., Jensen, E. L. N., \& Miller, N. A. 2008, MNRAS, 386, 1855

Coleiro, A., \& Chaty, S. 2013, ApJ, 764, 185

Cox, N. L. J., Kaper, L., \& Mokiem, M. R. 2005, A\&A, 436, 661

Cranmer, S. R., \& Owocki, S. P. 1996, ApJ, 462, 469

Davidson, K., \& Ostriker, J. P. 1973, ApJ, 179, 585

Drave, S. P., Clark, D. J., Bird, A. J., et al. 2010, MNRAS, 409, 1220

Drave, S. P., Bird, A. J., Sidoli, L., et al. 2013, MNRAS, 433, 528

Drave, S. P., Bird, A. J., Sidoli, L., et al. 2014, MNRAS, 439, 2175

Drew, J. E., Hoare, M. G., \& Denby, M. 1994, MNRAS, 266, 917

Farrell, S. A., Sood, R. K., O’Neill, P. M., \& Dieters, S. 2008, MNRAS, 389, 608

Feldmeier, A., \& Nikutta, R. 2006, A\&A, 446, 661

Feldmeier, A., \& Shlosman, I. 2000, ApJ, 532, L125

Feldmeier, A., Anzer, U., Boerner, G., \& Nagase, F. 1996, A\&A, 311, 793

Feldmeier, A., Puls, J., \& Pauldrach, A. W. A. 1997, A\&A, 322, 878

Feldmeier, A., Rätzel, D., \& Owocki, S. P. 2008, ApJ, 679, 704

Filliatre, P., \& Chaty, S. 2004, ApJ, 616, 469

Friend, D. B., \& Castor, J. I. 1982, ApJ, 261, 293

Gayley, K. G., Owocki, S. P., \& Cranmer, S. R. 1997, ApJ, 475, 786

Gies, D. R. 2003, in A Massive Star Odyssey: From Main Sequence to Supernova, eds. K. van der Hucht, A. Herrero, \& E. César (San Francisco: ASP), IAU Symp., 212, 91

González-Galán, A., Negueruela, I., Castro, N., et al. 2014, A\&A, 566, A131

Goossens, M. E., Bird, A. J., Drave, S. P., et al. 2013, MNRAS, 434, 2182

Hadrava, P., \& Čechura, J. 2012, A\&A, 542, A42

Hall, T. A., Finley, J. P., Corbet, R. H. D., \& Thomas, R. C. 2000, ApJ, 536, 450 Harmanec, P. 1988, Bull. Astron. Inst. Czechosl., 39, 329
Harvin, J. A., Gies, D. R., Bagnuolo, W. G., Jr., Penny, L. R., \& Thaller, M. L. 2002, ApJ, 565, 1216

Hatchett, S., \& McCray, R. 1977, ApJ, 211, 552

Herrero, A., Puls, J., \& Najarro, F. 2002, A\&A, 396, 949

Herrero, A., Kudritzki, R. P., Gabler, R., Vilchez, J. M., \& Gabler, A. 1995, A\&A, 297, 556

Higginbottom, N., Proga, D., Knigge, C., et al. 2014, ApJ, 789, 19

Hill, A. B., Walter, R., Knigge, C., et al. 2005, A\&A, 439, 255

Hoyle, F., \& Lyttleton, R. A. 1941, MNRAS, 101, 227

Hummer, D. G., Berrington, K. A., Eissner, W., et al. 1993, A\&A, 279, 298

Hung, L.-W., Hickox, R. C., Boroson, B. S., \& Vrtilek, S. D. 2010, ApJ, 720, 1202

Kaper, L., Hammerschlag-Hensberge, G., \& van Loon, J. T. 1993, A\&A, 279, 485

Kaper, L., Hammerschlag-Hensberge, G., \& Zuiderwijk, E. J. 1994, A\&A, 289, 846

Krtička, J. 2014, A\&A, 564, A70

Krtička, J., \& Kubát, J. 2004, A\&A, 417, 1003

Krtička, J., \& Kubát, J. 2009, MNRAS, 394, 2065

Krtička, J., \& Kubát, J. 2010, A\&A, 519, A5

Krtička, J., \& Kubát, J. 2011, A\&A, 534, A97

Krtička, J., \& Kubát, J. 2012, MNRAS, 427, 84

Krtička, J., Kubát, J., \& Skalický, J. 2012, ApJ, 757, 162

Krtička, J., Kurfürst, P., \& Krtičková, I. 2015, A\&A, 573, A20

Kubát, J. 2003, in Modelling of Stellar Atmospheres, eds. N. E. Piskunov, W. W.

Weiss, \& D. F. Gray (San Francisco: ASP), IAU Symp., 210, A8

Kubát, J., Puls, J., \& Pauldrach, A. W. A. 1999, A\&A, 341, 587

Kupka, F., Piskunov, N. E., Ryabchikova, T. A., Stempels, H. C., \& Weiss, W. W. 1999, A\&AS, 138, 119

Lamers, H. J. G. L. M., \& Snow, T. P. 1978, ApJ, 219, 504

Lamers, H. J. G. L. M., van den Heuvel, E. P. J., \& Petterson, J. A. 1976, A\&A, 49,327

Lamers H. J. G. L. M., Snow T. P., \& Lindholm D. M. 1995, ApJ, 455, 269

Lanz, T., \& Hubeny, I. 2003, ApJS, 146, 417

Lanz, T., \& Hubeny, I. 2007, ApJS, 169, 83

La Parola, V., Cusumano, G., Romano, P., et al. 2010, MNRAS, 405, L66

Leung, K.-C., Moffat, A. F. J., \& Seggewiss, W. 1979, ApJ, 231, 742

Li, T., \& Li, X.-D. 2014, ApJ, 780, 47

Linder, N., Rauw, G., Pollock, A. M. T., \& Stevens, I. R. 2006, MNRAS, 370, 1623

Linder, N., Rauw, G., Martins, F., et al. 2008, A\&A, 489, 713

Lorenzo, J., Negueruela, I., Castro, N., et al. 2014, A\&A, 562, A18

Lucy, L. B. 2012, A\&A, 544, A120

Lucy L. B., \& White, R. L. 1980, ApJ, 241, 300

MacGregor, K. B., \& Vitello, P. A. J. 1982, ApJ, 259, 267

MacFarlane, J. J., Cohen, D. H., \& Wang, P. 1994, ApJ, 437, 351

Mahy, L., Martins, F., Machado, C., Donati, J.-F., \& Bouret, J.-C. 2011, A\&A, 533, A9

Mahy, L., Gosset, E., Sana, H., et al. 2012, A\&A, 540, A97

Manousakis, A., Walter, R., \& Blondin, J. M. 2012, A\&A, 547, A20

Marchenko, S. V., Rauw, G., Antokhina, E. A., et al. 2000, MNRAS, 317, 333

Martins, F., Schaerer, D., \& Hillier, D. J. 2005a, A\&A, 436, 1049

Martins, F., Schaerer, D., Hillier, D. J., et al. 2005b, A\&A, 441, 735

Mason, A. B., Norton, A. J., Clark, J. S., Negueruela, I., \& Roche, P. 2011a, A\&A, 532, A124

Mason, A. B., Norton, A. J., Clark, J. S., Roche, P., \& Negueruela, I. 2011b, Bull. Soc. Roy. Sci. Liège, 80, 699

Mason, A. B., Clark, J. S., Norton, A. J., et al. 2012, MNRAS, 422, 199

Mayer, P., Harmanec, P., Nesslinger, S., et al. 2008, A\&A, 481, 183

Mayer, P., Harmanec, P., Wolf, M., Božić, H., \& Šlechta, M. 2010, A\&A, 520, A89

McSwain, M. V., Gies, D. R., Huang, W., et al. 2004, ApJ, 600, 927

Mihalas, D., Kunasz, P. B., \& Hummer, D. G. 1975, ApJ, 202, 465

Moldón, J., Ribó, M., \& Paredes, J. M. 2012, A\&A, 548, A103

Montes, G., Ramirez-Ruiz, E., De Colle, F., \& Strickler, R. 2013, ApJ, 777, 129

Najarro, F., Hanson, M. M., \& Puls, J. 2011, A\&A, 535, A32

Naik, S., Paul, B., \& Ali, Z. 2011, ApJ, 737, 79

Negueruela, I., \& Coe, M. J. 2002, A\&A, 385, 517

Orosz, J. A., Steeghs, D., McClintock, J. E., et al. 2009, ApJ, 697, 573

Oskinova, L. M., Feldmeier, A., \& Kretschmar, P. 2012, MNRAS, 421, 2820

Owocki, S. P., \& Rybicki, G. B. 1984, ApJ, 284, 337

Owocki, S. P., Castor, J. I., \& Rybicki, G. B. 1988, ApJ, 335, 914

Pachoulakis, I. 1996, MNRAS, 280, 153

Parkin, E. R., \& Sim, S. A. 2013, ApJ, 767, 114

Parkin, E. R., Broos, P. S., Townsley, L. K., et al. 2011, ApJS, 194, 8

Pauldrach, A. W. A., \& Puls, J. 1990, A\&A, 237, 409

Pauldrach, A. W. A., Kudritzki, R. P., Puls, J., Butler, K., \& Hunsinger, J. 1994, A\&A, 283, 525 
J. Krtička et al.: The effect of the X-ray irradiation on the wind in binaries with hot component

Pauldrach, A. W. A., Hoffmann, T. L., \& Lennon, M. 2001, A\&A, 375, 161 Pellizza, L. J., Chaty, S., \& Negueruela, I. 2006, A\&A, 455, 653

Penny, L. R., Seyle, D., Gies, D. R., et al. 2001, ApJ, 548, 889

Piskunov, N. E., Kupka, F., Ryabchikova, T. A., Weiss, W. W., \& Jeffery, C. S. 1995, A\&AS, 112, 525

Pittard, J. M. 2009, MNRAS, 396, 1743

Porter, J. M., \& Skouza, B. A. 1999, A\&A, 344, 205

Prat, L., Rodriguez, J., Hannikainen, D. C., \& Shaw, S. E. 2008, MNRAS, 389, 301

Prilutskii, O. F., \& Usov, V. V. 1976, AZh, 53, 6

Rahoui, F., \& Chaty, S. 2008, A\&A, 492, 163

Rauw, G., Nazé, Y., Carrier, F., et al. 2001, A\&A, 368, 212

Rauw, G., Crowther, P. A., De Becker, M., et al. 2005, A\&A, 432, 985

Rauw, G., Sana, H., Spano, M., et al. 2012, A\&A, 542, A95

Rawls, M. L., Orosz, J. A., McClintock, J. E., et al. 2011, ApJ, 730, 25

Reig, P. 2011, Ap\&SS, 332, 1

Reig, P., Chakrabarty, D., Coe, M. J., et al. 1996, A\&A, 311, 879

Ribó, M., Negueruela, I., Blay, P., Torrejón, J. M., \& Reig, P. 2006, A\&A, 449, 687

Romano, P., Sidoli, L., Mangano, V., Mereghetti, S., \& Cusumano, G. 2007, A\&A, 469, L5

Romano, P., Sidoli, L., Ducci, L., et al. 2010, MNRAS, 401, 1564

Rybicki, G. B., \& Hummer, D. G. 1978, ApJ, 219, 645

Sahade, J., \& Brandi, E. 1991, ApJ, 379, 706

Sana, H., Stevens, I. R., Gosset, E., Rauw, G., \& Vreux, J.-M. 2004, MNRAS, 350,809

Sana, H., Antokhina, E., Royer, P., et al. 2005, A\&A, 441, 213

Sana, H., Gosset, E., \& Rauw, G. 2006a, MNRAS, 371, 67

Sana, H., Rauw, G., Nazé, Y., Gosset, E., Vreux, J.-M. 2006b, MNRAS, 372, 661

Sana, H., Nazé, Y., O’Donnell, B., Rauw, G., \& Gosset, E. 2008, New Astron., 13,202

Sana, H., Le Bouquin, J.-B., Mahy, L., et al. 2013, A\&A, 553, A131

Sanchez-Bermudez, J., Schödel, R., Alberdi, A., et al. 2013, A\&A, 554, L4
Searle, S. C., Prinja, R. K., Massa, D., \& Ryans, R. 2008, A\&A, 481, 777 Seaton, M. J., Zeippen, C. J., Tully, J. A., et al. 1992, Rev. Mex. Astron. Astrofis., 23, 19

Sguera, V. 2013, Nucl. Phys. B Proc. Supp., 239, 76

Sguera, V., Bird, A. J., Dean, A. J., et al. 2007, A\&A, 462, 695

Sguera, V., Drave, S. P., Bird, A. J., et al. 2011, MNRAS, 417, 573

Sidoli, L., Esposito, P., Sguera, V., et al. 2013, MNRAS, 429, 2763

Sierpowska-Bartosik, A., \& Bednarek, W. 2008, MNRAS, 385, 2279

Simón-Díaz, S., Caballero, J. A., \& Lorenzo, J. 2011, ApJ, 742, 55

Snow, T. P., \& Morton, D. C. 1976, ApJS, 32, 429

Stickland, D. J. 1995, The Observatory, 115, 180

Straižys, V., \& Kuriliene, G. 1981, Ap\&SS, 80, 353

Sturm, E., \& Simon, K. P. 1994, A\&A, 282, 93

Suchy, S., Pottschmidt, K., Wilms, J., et al. 2008, ApJ, 675, 1487

Sushch, I., de Naurois, M., Schwanke, U., Spengler, G., \& Bordas, P. 2013, 2012 Fermi Symp. Proc. - eConf C121028 [arXiv: 1303.1316]

Tarter, C. B., Tucker, W. H., \& Salpeter, E. E. 1969, ApJ, 156, 943

ten Brummelaar, T. A., O’Brien, D. P., Mason, B. D., et al. 2011, AJ, 142, 21

Thompson, T. W. J., Tomsick, J. A., in 't Zand, J. J. M., Rothschild, R. E., \& Walter, R. 2007, ApJ, 661, 447

Tomsick, J. A., Chaty, S., Rodriguez, J., et al. 2009, ApJ, 694, 344

Tomsick, J. A., \& Muterspaugh, M. W. 2010, ApJ, 719, 958

ud-Doula, A., Owocki, S., Townsend, R., Petit, V., \& Cohen, D. 2014, MNRAS, 441,3600

Val Baker, A. K. F., Norton, A. J., \& Quaintrell, H. 2005, A\&A, 441, 685

van Kerkwijk, M. H., van Paradijs, J., \& Zuiderwijk, E. J. 1995, A\&A, 303, 483

van Loon, J. Th., Kaper, L., \& Hammerschlag-Hensberge, G. 2001, A\&A, 375, 498

van der Meer, A., Kaper, L., di Salvo, T., et al. 2005, A\&A, 432, 999

Vink, J. S., de Koter, A., \& Lamers, H. J. G. L. M. 1999, A\&A, 350, 181

Vink, J. S., de Koter, A., \& Lamers, H. J. G. L. M. 2000, A\&A, 362, 295

Yan, J., Liu, Q., \& Hadrava, P. 2008, AJ, 136, 631

Watanabe, S., Sako, M., Ishida, M., et al. 2006, ApJ, 651, 421

Zurita Heras, J. A., \& Walter, R. 2009, A\&A, 494, 1013

Pages 12 to 15 are available in the electronic edition of the journal at http://www . aanda. org 
A\&A 579, A111 (2015)
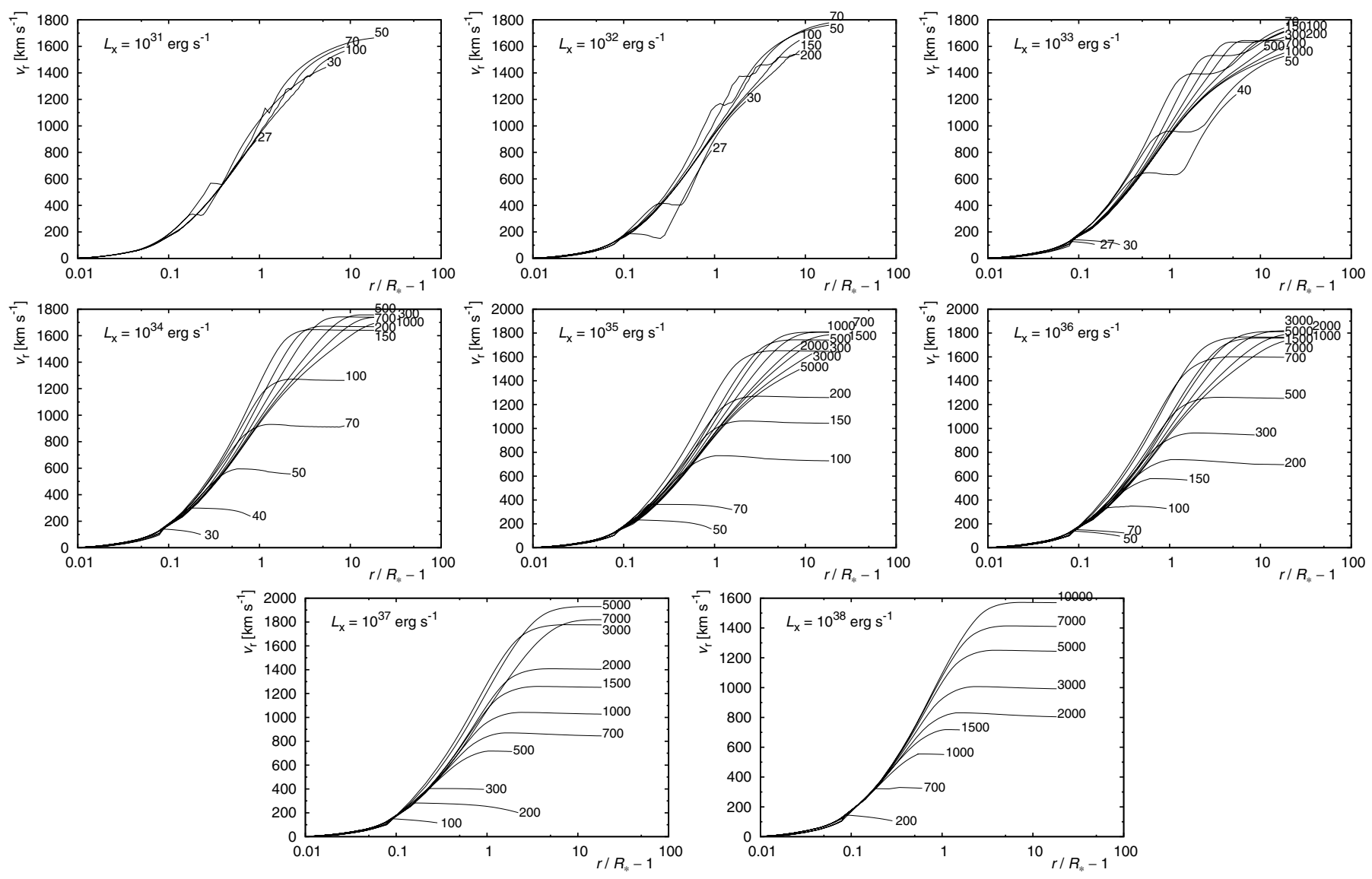

Fig. 3. Dependence of the radial velocity on the radius in the wind of 300-1 star model. Each graph corresponds to different luminosity of additional X-ray source $L_{\mathrm{X}}$ labelled in the graph. Each radial velocity plot is labelled with a corresponding X-ray source distance $D$ in $R_{\odot}$. 
J. Krtička et al.: The effect of the X-ray irradiation on the wind in binaries with hot component
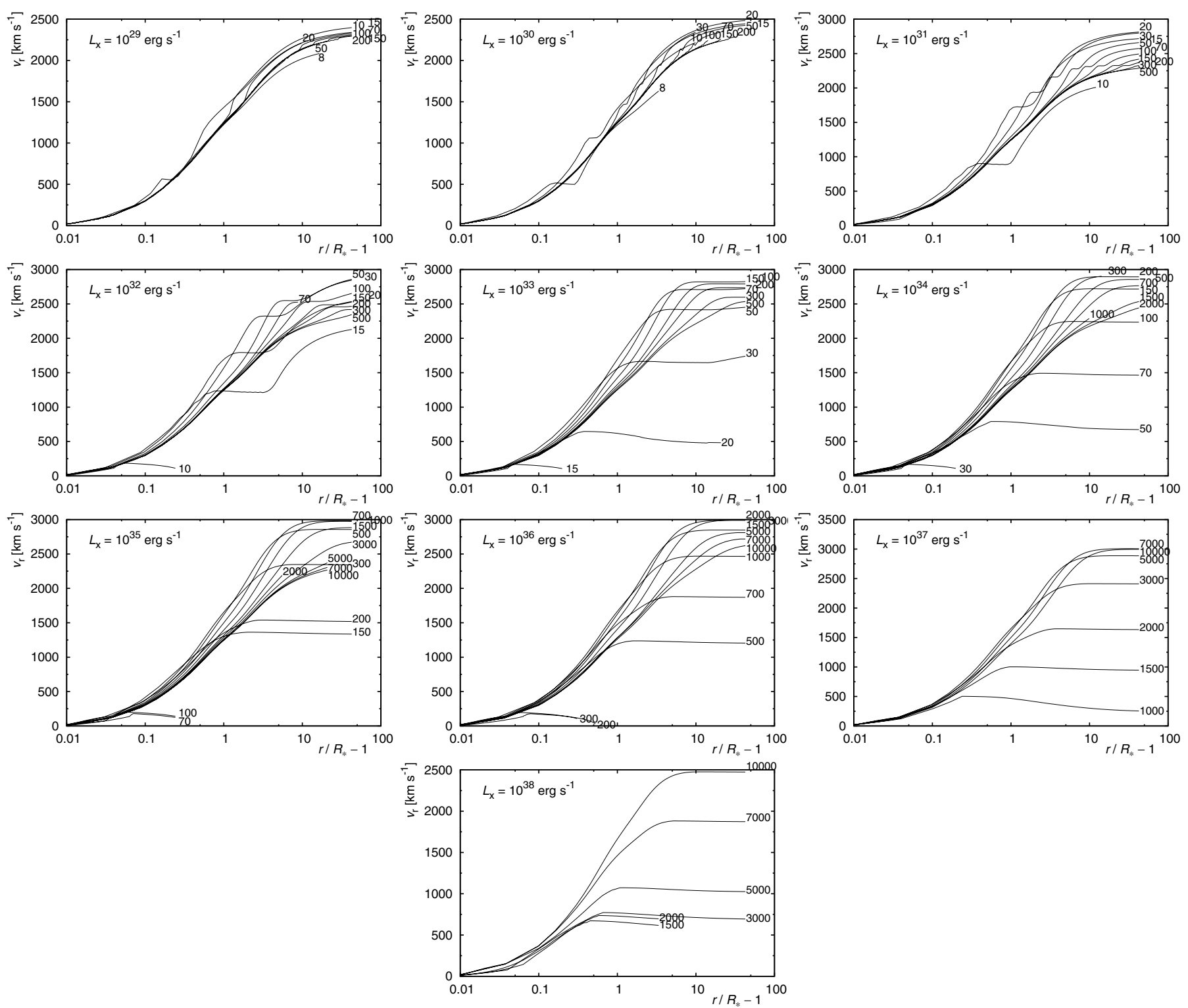

Fig. 4. Same as Fig. 3, except for a model star 300-5. 
A\&A 579, A111 (2015)
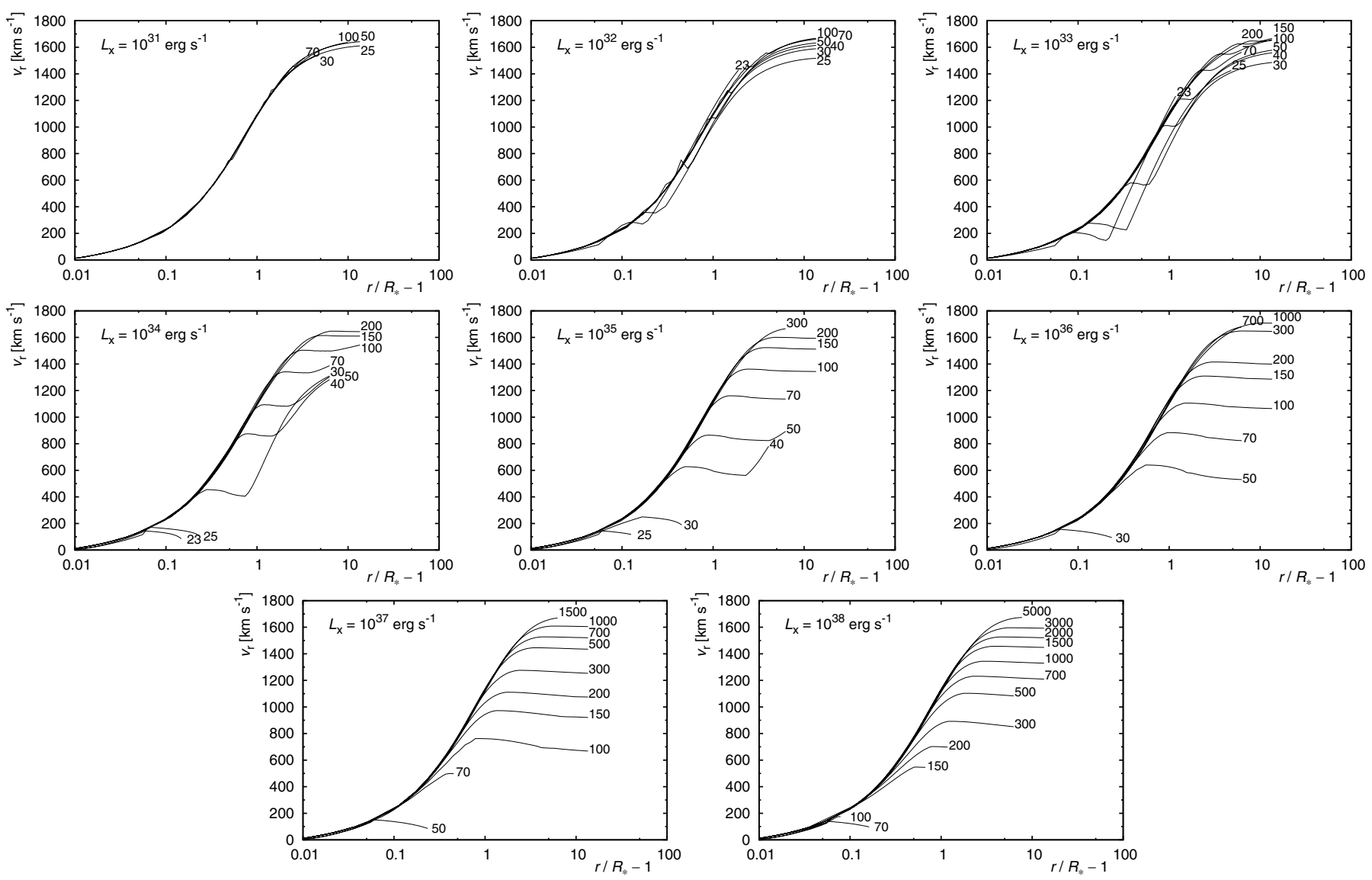

Fig. 5. Same as Fig. 3, except for a model star 375-1.
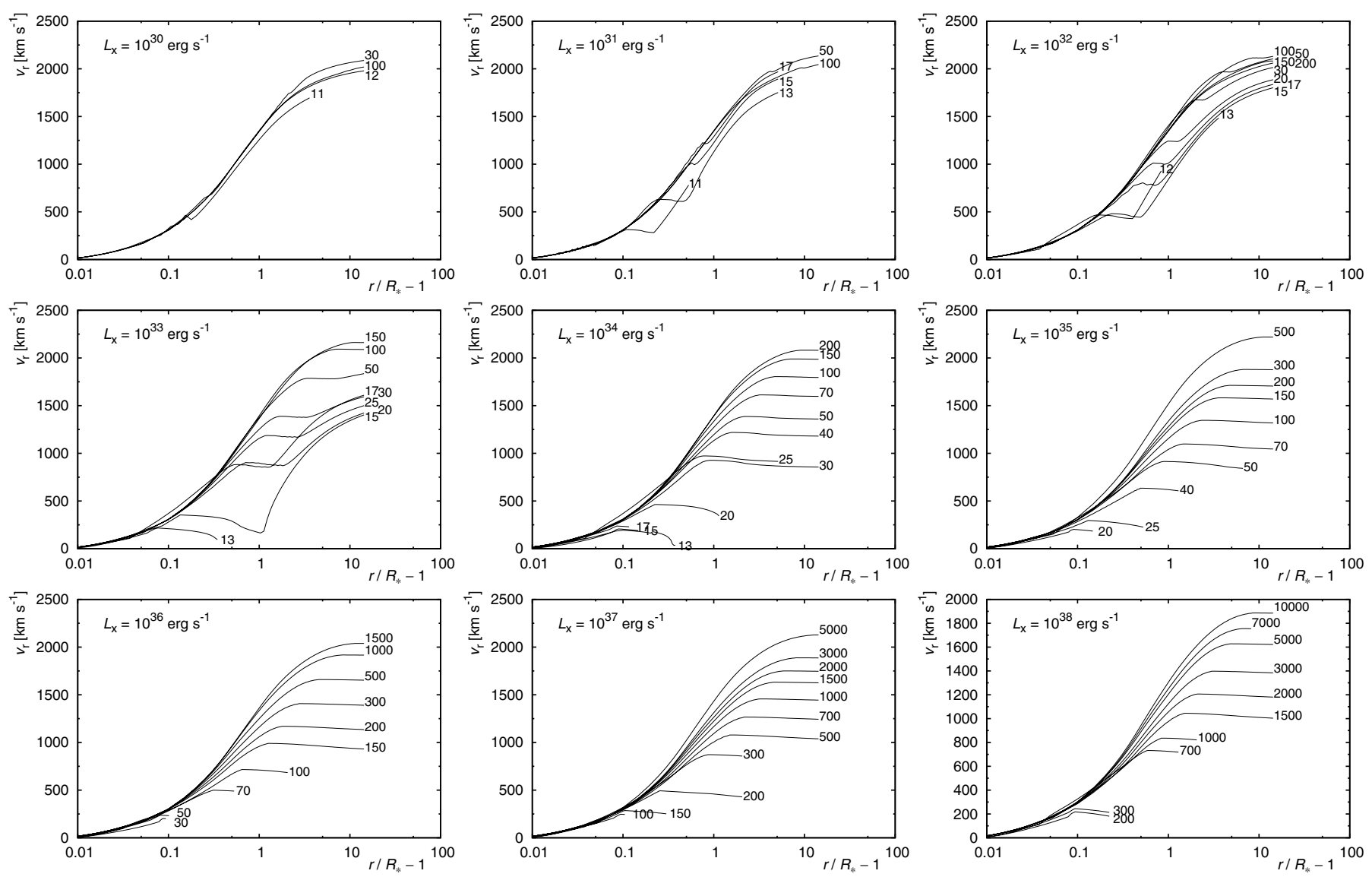

Fig. 6. Same as Fig. 3, except for a model star 375-5. 
J. Krtička et al.: The effect of the X-ray irradiation on the wind in binaries with hot component
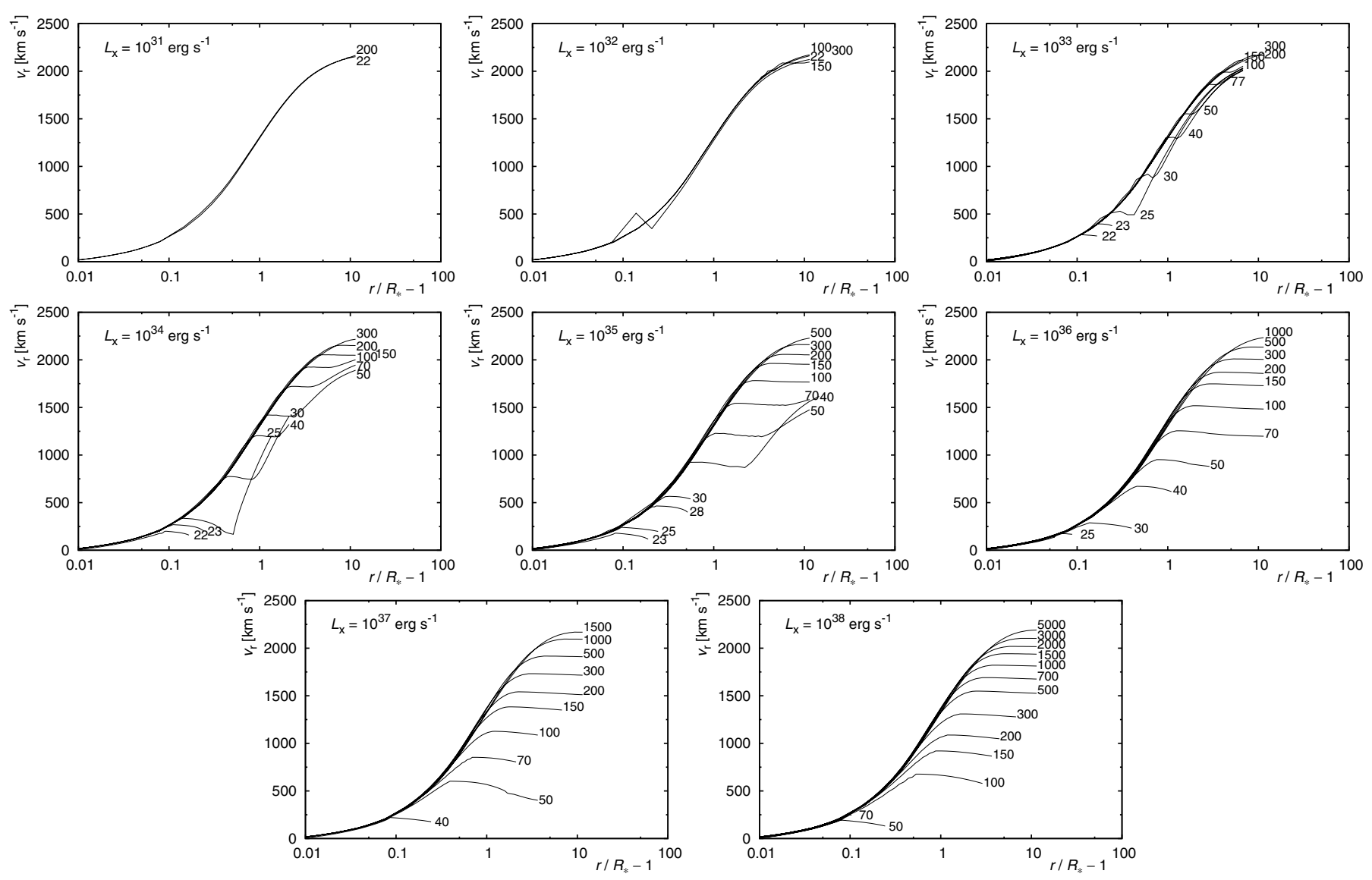

Fig. 7. Same as Fig. 3, except for a model star 425-1.
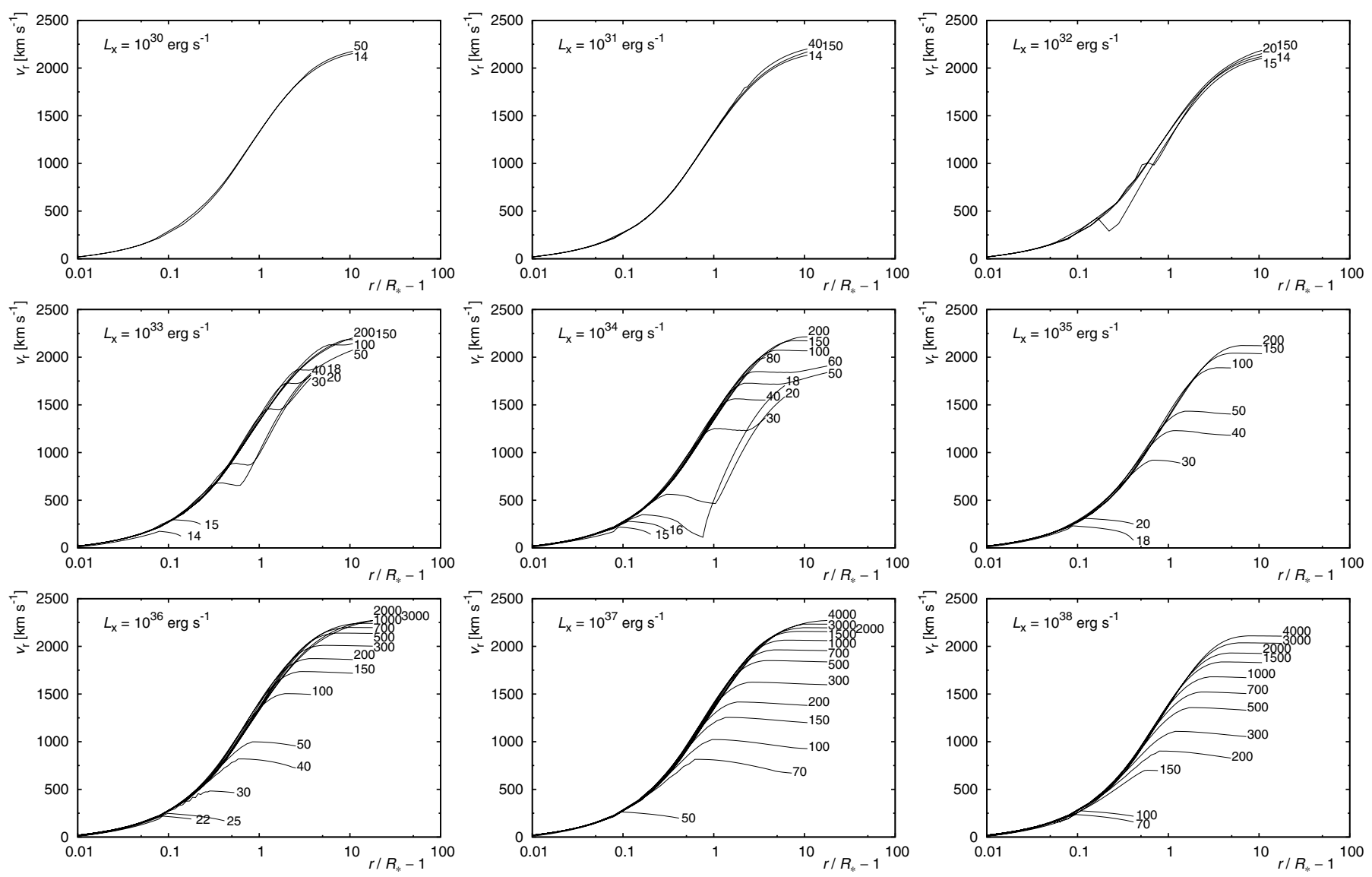

Fig. 8. Same as Fig. 3, except for a model star 425-5. 